\title{
S. Visweswaran
}

\section{Some remarks on multiplicatively closed sets}

Received: 14 November 2012 / Accepted: 22 May 2013 / Published online: 12 June 2013

(C) The Author(s) 2013. This article is published with open access at Springerlink.com

\begin{abstract}
Rings considered in this article are commutative with identity. A subring of a ring is assumed to contain the identity element of the ring. Let $S$ be a multiplicatively closed subset of a ring $R$ satisfying the following property $(P)$ : whenever $a b \in S$ with at least one of $a, b$ is in $S$, then both of them are in $S$. The $(P)$ closure of any multiplicatively closed subset of $R$ is discussed; and saturated multiplicatively closed subsets of the form $1+I$, where $I$ is an ideal of $R$ are also considered. We investigate the question of determining rings admitting only a finite number of such multiplicatively closed sets. In addition, we discuss the problem of whether a ring $R$ admitting only a finite number of saturated multiplicatively closed subsets of the form $1+I$, where $I$ is an ideal of $R$, extends to the polynomial ring $R[x]$ in one variable $x$ over $R$.
\end{abstract}

\section{Mathematics Subject Classification 13Axx}

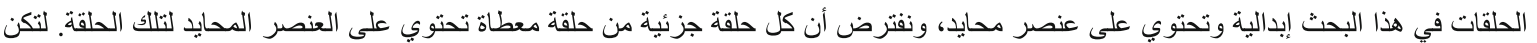

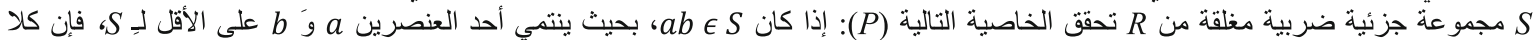

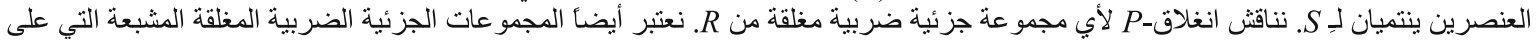

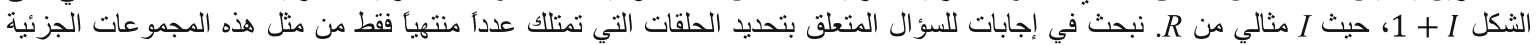

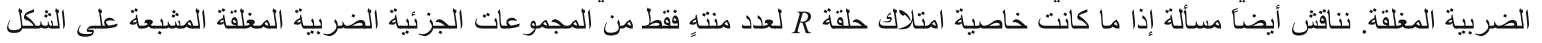

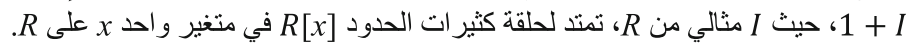

\section{Introduction}

Let $R$ be a commutative ring with identity. Recall from [1, p. 36] that a subset $S$ of $R$ is said to be a multiplicatively closed subset of $R$ if: $1 \in S$ and for any $s_{1}, s_{2} \in S, s_{1} s_{2} \in S$. This article is motivated by [1, Problem 7, p. 44]. Recall from [1, Problem 7, p. 44] that a multiplicatively closed subset $S$ of $R$ is said to be saturated if the following holds: for any $x, y \in R, x y \in S$ if and only if $x \in S$ and $y \in S$. The authors of [1] asked to prove in [1, Problem 7(i), p. 44] that a multiplicatively closed subset $S$ of $R$ is saturated if and only if $R \backslash S$ is a union of prime ideals. Moreover, in [1, Problem 7(ii), p. 44], the readers are asked to prove that given any multiplicatively closed subset $S$ of $R$, there exists a unique smallest saturated multiplicatively closed subset $\bar{S}$ containing $S$ and that $\bar{S}$ is the complement in $R$ of the union of the prime ideals of $R$ which do not meet $S$. Furthermore, the readers are asked to find $\bar{S}$ where $S=1+I$, that is, $S=\{1+a \mid a \in I\}$ for some ideal $I$ of $R$. It follows from [1, Problem 7(ii), p. 44] that if $I$ is any ideal of $R$ and if $S=1+I$, then $\bar{S}=R \backslash\left(\cup_{P \in \Delta} P\right)$ where $\Delta=\{P \mid \mathrm{P}$ is a prime ideal of $R$ such that $P \cap(1+I)=\emptyset\}$.

S. Visweswaran $(\varangle)$

Department of Mathematics, Saurashtra University, Rajkot 360 005, India

E-mail: s_visweswaran2006@yahoo.co.in 
Observe that $P \in \Delta$ if and only if $1 \notin P+I$ if and only if $P+I \neq R$. It bas been pointed to me by the referee that if $\Delta_{1}=\{M \in \Delta \mid \mathrm{M}$ is a maximal ideal of $R\}$, then $\bar{S}=R \backslash\left(\cup_{M \in \Delta_{1}} M\right)$. Since $\Delta_{1} \subseteq \Delta$, it is clear that $\cup_{M \in \Delta_{1}} M \subseteq \cup_{P \in \Delta} P$. Let $P \in \Delta$. Then $P+I \neq R$. Hence, there exists a maximal ideal $M$ of $R$ such that $P+I \subseteq M$. Now, $M$ is a prime ideal of $R, M+I \neq R$ and so $M \in \Delta$. Thus, $M \in \Delta_{1}$ and as $P \subseteq M$, it follows that $\cup_{P \in \Delta} P \subseteq \cup_{M \in \Delta_{1}} M$. This proves that $\cup_{P \in \Delta} P=\cup_{M \in \Delta_{1}} M$ and so $\bar{S}=R \backslash\left(\cup_{M \in \Delta_{1}} M\right)$. However, if $I$ is any ideal of $R$, then $1+I$ has the following property: whenever $a b \in 1+I$ for some $a, b \in R$ and if either $a$ or $b$ belongs to $1+I$, then both $a$ and $b$ belong to $1+I$ (for a proof see Example 2.1(i)).

The observations mentioned in the previous paragraph motivate the following definition. Let $R$ be a ring and let $S$ be a multiplicatively closed subset of $R$. We say that $S$ satisfies property $(P)$ if the following holds: whenever $a b \in S$ for some $a, b \in R$ and if either $a$ or $b$ belongs to $S$, then both of them belong to $S$. In such a case, we simply say that $S$ satisfies $(P)$.

Let $R$ be a ring. In Sect. 2 of this article, we provide some examples of multiplicatively closed subsets $S$ of $R$ that satisfy $(P)$. It is noted in Remark 2.2(ii) that the multiplicatively closed subset $Z(R) \cup\{1\}$ satisfies $(P)$ if and only if $Z(R) \cup\{1\}=R$, where $Z(R)$ denotes the set of all zero-divisors of $R$. In the rest of Sect. 2, we attempt to characterize rings $R$ such that $Z(R) \cup\{1\}=R$. Though I do not know any characterization of a ring $R$ with the property $Z(R) \cup\{1\}=R$, however, under some additional hypotheses on $R$, we characterize $R$ such that $Z(R) \cup\{1\}=R$ (see Propositions 2.7 and 2.11). We provide in Proposition 2.12, a necessary and sufficient condition in order that a multiplicatively closed subset $S$ of a ring $R$ to satisfy $(P)$.

Let $S$ be a multiplicatively closed subset of a ring $R$. Note that $S_{1}=\cap_{T \supseteq S} T$, where $T$ varies over all multiplicatively closed subsets of $R, T \supseteq S$, and $T$ satisfies $(P)$ is the smallest multiplicatively closed subset of $R$ such that $S_{1} \supseteq S$ and $S_{1}$ satisfies $(P)$. We call $S_{1}$, the $(P)$-closure of $S$ and we denote it by $(P)-\operatorname{cl}(S)$. In Proposition 3.2, we characterize $(P)-\operatorname{cl}(S)$ in terms of its elements. Let $T$ be a ring and let $R$ be a subring of $T$. Let $S$ be a multiplicatively closed subset of $R$. We call the smallest multiplicatively closed subset of $T$ containing $S$ and which satisfies $(P)$, the $(P)$-closure of $S$ in $T$ and denote it by $(P)-\operatorname{cl}_{T}(S)$. Under the hypothesis that $T$ is an integral domain, in the rest of Sect. 3, we determine $(P)-\operatorname{cl}_{T}(S)$ for some multiplicatively closed subsets $S$ of $R$.

Let $I$ be an ideal of a ring $R$. In Sect. 4, we focus our study on ideals $I$ such that the multiplicatively closed subset $1+I$ of $R$ is saturated. We prove in Proposition 4.1 that if $I$ is an ideal of $\mathbf{Z}$, then $1+I$ is saturated if and only if either $I=\mathbf{Z}$ or $I=2 \mathbf{Z}$. Motivated by the assertion of Proposition 4.1, we attempt in this section to provide classes of rings $R$ such that $R$ admits only finitely many saturated multiplicatively closed subsets of the form $1+I$, where $I$ is an ideal of $R$ (see Propositions 4.3(i) and 4.5). We also provide some examples to illustrate the results proved in Sect. 4.

For any ring $R$, let $R[x]$ (respectively $R[[x]]$ ) denote the polynomial (respectively the power series) ring in one variable $x$ over $R$. We denote by $\mathcal{R}$, the collection of all rings $R$ with the property that $R$ admits only a finite number of saturated multiplicatively closed subsets of the form $1+I$, where $I$ is an ideal of $R$. The main problem discussed in Sect. 5 is to determine whether a ring $R \in \mathcal{R}$ implies that $R[x] \in \mathcal{R}$ (respectively $R[[x]] \in \mathcal{R}$ ). We prove in Proposition 5.3 that $R \in \mathcal{R}$ if and only if $R[[x]] \in \mathcal{R}$. I do not know whether $R \in \mathcal{R}$ implies that $R[x] \in \mathcal{R}$. However, we prove under some additional hypotheses on $R$ that if $R \in \mathcal{R}$, then $R[x] \in \mathcal{R}$.

\section{Examples of multiplicatively closed subsets with property $(P)$}

In this section, we give some examples of multiplicatively closed subsets of a ring $R$ that satisfy $(P)$. We begin with the following example:

Example 2.1 Let $R$ be a ring.

(i) If $I$ is any ideal of $R$, then the multiplicatively closed subset $S=1+I$ of $R$ satisfies $(P)$.

(ii) Let $Z(R)$ denote the set of all zero-divisors of $R$. If $a \notin Z(R) \cup U(R)$, then the multiplicatively closed subset $S=\left\{a^{n} \mid n \geq 0\right\}$ of $R$ satisfies $(P)$. In particular, for any $a \in \mathbf{Z} \backslash\{0,1,-1\}, S=\left\{a^{n} \mid n \geq 0\right\}$ satisfies $(P)$.

(iii) The multiplicatively closed subset $S=\left\{2^{n} \mid n \geq 0\right\}$ of $\mathbf{Z}$ satisfies $(P)$, but $S \neq 1+I$ for any ideal $I$ of $\mathbf{Z}$.

(iv) Let $R[x]$ denote the polynomial ring in one variable over $R$. Let $S=\{f(x) \in R[x] \mid f(x)$ is monic $\}$. Then $S$ is a multiplicatively closed subset of $R[x], S$ satisfies $(P)$ but $S \neq 1+I$ for any ideal $I$ of $R[x]$.

Verification. (i) Let $a, b \in R$ be such that $a b \in S=1+I$. Assume that $a \in 1+I$. Now $a=1+x$ for some $x \in I$ and $a b=1+y$ for some $y \in I$. Hence, $a b=(1+x) b=1+y$. Observe that $y-x b \in I$ and therefore, $b=1+(y-x b) \in 1+I$.

The assertions (ii), (iii), and (iv) can be easily verified and hence their verifications are omitted. 
We mention in Example 2.3, a multiplicatively closed subset of a ring which does not satisfy the property $(P)$ with the help of the following remark.

Remark 2.2 Let $R$ be a ring.

(i) Let $S$ be a multiplicatively closed subset of $R$ with $0 \in S$. Then $S$ satisfies $(P)$ if and only if $S=R$.

(ii) Let $S=Z(R) \cup\{1\}$. Then $S$ satisfies $(P)$ if and only if $Z(R) \cup\{1\}=R$.

Proof The proof of this remark is obvious and hence is omitted.

Example 2.3 Let $R=\mathbf{Z} / 6 \mathbf{Z}$. Note that $Z(R)=\{0+6 \mathbf{Z}, 2+6 \mathbf{Z}, 4+6 \mathbf{Z}, 3+6 \mathbf{Z}\}$. Let $S=Z(R) \cup\{1+6 \mathbf{Z}\}$. Note that $5+6 \mathbf{Z} \notin S$. Hence $S \neq \mathbf{Z} / 6 \mathbf{Z}$. Therefore, $S$ does not satisfy $(P)$. Indeed, $(2+6 \mathbf{Z})(5+6 \mathbf{Z})=4+6 \mathbf{Z} \in S$ and $2+6 \mathbf{Z} \in S$ but $5+6 \mathbf{Z} \notin S$.

The following remark provides examples of multiplicatively closed subsets that do not satisfy $(P)$ and which do not contain the zero element of the ring.

Remark 2.4 Let $R$ be an integral domain which is not a field. Let $I$ be a nonzero ideal of $R$ with $I \neq R$. Let $S=(I \backslash\{0\}) \cup\{1\}$. Then $S$ is a multiplicatively closed subset of $R$ and $S$ does not satisfy $(P)$. In particular, if $n \in \mathbf{Z}$ with $n \geq 2$, then $S=(n \mathbf{Z} \backslash\{0\}) \cup\{1\}$ is a multiplicatively closed subset of $\mathbf{Z}$ and $S$ does not satisfy $(P)$.

Proof The proof of this remark is quite obvious and hence is omitted.

It is noted in Remark 2.2(ii) that the multiplicatively closed subset $S=Z(R) \cup\{1\}$ of a ring $R$ satisfies $(P)$ if and only if $Z(R) \cup\{1\}=R$. It is natural to consider the following question.

Q. What are the necessary and sufficient conditions on a ring $R$ in order that $R$ satisfies $Z(R) \cup\{1\}=R$ ?

In the following remarks, we attempt to answer the above question. Note that $Z(R) \cup\{1\}=R$ implies that $U(R)=\{1\}$.

Remark 2.5 Let $R$ be a ring. If $U(R)=\{1\}$, then the following hold:

(i) $r+r=0$ for all $r \in R$.

(ii) $R$ is reduced. That is, $R$ has no nonzero nilpotent elements.

(iii) $J(R)=(0)$ where $J(R)$ denotes the Jacobson radical of $R$.

Proof Note that $-1 \in U(R)=\{1\}$ and so $-1=1$. Hence $1+1=0$ and so $r+r=0$ for all $r \in R$.

(ii) Let $a$ be a nilpotent element of $R$. Then $1+a \in U(R)$. Since $U(R)=\{1\}$, it follows that $1+a=1$ and this implies that $a=0$. This proves that $R$ is reduced.

(iii) Let $r \in J(R)$. Then $1+r \in U(R)$ by [1, Proposition 1.9]. Hence $1+r=1$ and so $r=0$. This proves that $J(R)=(0)$.

The following remark determines rings $R$ with the following properties: $Z(R)$ is an ideal of $R$ and $Z(R) \cup$ $\{1\}=R$.

Remark 2.6 Let $R$ be a ring. Suppose that $Z(R)$ is an ideal of $R$. Then $Z(R) \cup\{1\}=R$ if and only if $R$ is isomorphic to $\mathbf{Z} / 2 \mathbf{Z}$ as rings.

Proof Suppose that $Z(R) \cup\{1\}=R$. Let $a \in Z(R)$. By hypothesis, $Z(R)$ is an ideal of $R$. As $a \in Z(R)$ and $1 \notin Z(R)$, it follows that $1+a \notin Z(R)$. Hence $1+a \in R \backslash Z(R)=\{1\}$. Thus $1+a=1$ and so $a=0$. This shows that $Z(R)=(0)$ and hence from $Z(R) \cup\{1\}=R$, it follows that $R=\{0,1\}$ and so $R$ is isomorphic to $\mathbf{Z} / 2 \mathbf{Z}$ as rings.

Conversely, if $R$ is isomorphic to $\mathbf{Z} / 2 \mathbf{Z}$ as rings, then it is clear that $R=\{0,1\}=Z(R) \cup\{1\}$.

Let $R$ be a ring. Note that $S=R \backslash Z(R)$ is a saturated multiplicatively closed subset of $R$. Hence, we obtain from [1, Problem 7(i), p. 44] that $Z(R)=R \backslash S$ is a union of prime ideals of $R$. Moreover, if the zero ideal of $R$ admits a primary decomposition, then $Z(R)$ is the union of a finite number of prime ideals of $R[1$, Proposition 4.7]. In Proposition 2.7, we determine rings $R$ such that $Z(R) \cup\{1\}=R$ under the assumption that $Z(R)$ is the union of a finite number of prime ideals of $R$.

Proposition 2.7 Let $P_{i}(i=1, \ldots, n)$ be prime ideals of a ring $R$ such that $Z(R)=\cup_{i=1}^{n} P_{i}$. Then $Z(R) \cup$ $\{1\}=R$ if and only if $R$ is isomorphic to $F_{1} \times \cdots \times F_{m}$ as rings for some $m \geq 1$, where $F_{i}=\mathbf{Z} / 2 \mathbf{Z}$ for $i=1, \ldots, m$.

Proof Suppose that $Z(R) \cup\{1\}=R$. Since $Z(R)=\cup_{i=1}^{n} P_{i}$, it follows that $\left(\cup_{i=1}^{n} P_{i}\right) \cup\{1\}=R$. From $Z(R) \cup\{1\}=R$, it follows that if $I$ is any proper ideal of $R$, then $I \subseteq Z(R)$. For each $i \in\{1, \ldots, n\}$ let $M_{i}$ be a maximal ideal of $R$ such that $P_{i} \subseteq M_{i}$. Now $Z(R)=\cup_{i=1}^{n} P_{i} \subseteq \cup_{i=1}^{n} M_{i} \subseteq Z(R)$. Hence, 
we obtain that $Z(R)=\cup_{i=1}^{n} M_{i}$. We may assume without loss of generality that $M_{1}, \ldots, M_{m}$ are distinct among $M_{1}, M_{2}, \ldots, M_{n}$. Note that $Z(R)=\cup_{i=1}^{m} M_{i}$. We assert that $\left\{M_{1}, \ldots, M_{m}\right\}$ is the set of all maximal ideals of $R$. Let $M$ be any maximal ideal of $R$. Then $M \subseteq Z(R)=\cup_{i=1}^{m} M_{i}$. This implies in view of [1, Proposition 1.11(i)] that $M \subseteq M_{i}$ for some $i \in\{1, \ldots, m\}$ and so $M=M_{i}$. This shows that $\left\{M_{1}, \ldots, M_{m}\right\}$ is the set of all maximal ideals of $R$. Since $Z(R) \cup\{1\}=R$, we obtain that $U(R)=\{1\}$. Hence it follows from Remark 2.5(iii) that $\cap_{i=1}^{m} M_{i}=(0)$. Now we obtain from the Chinese remainder theorem [1, Proposition 1.10(ii)] that the mapping $f: R \rightarrow R / M_{1} \times \cdots \times R / M_{m}$ given by $f(r)=\left(r+M_{1}, \ldots, r+M_{m}\right)$ is an isomorphism of rings. Since $U(R)=\{1\}$, it follows that $U\left(R / M_{1} \times \cdots \times R / M_{m}\right)=\left\{\left(1+M_{1}, \ldots, 1+M_{m}\right)\right\}$. As $U\left(R / M_{1} \times \cdots \times R / M_{m}\right)=U\left(R / M_{1}\right) \times \cdots \times U\left(R / M_{m}\right)$, it follows that $U\left(R / M_{i}\right)=\left\{1+M_{i}\right\}$ for $i=1, \ldots, m$, and since $R / M_{i}$ is a field, we obtain that $R / M_{i}=\left\{0+M_{i}, 1+M_{i}\right\}$ for $i=1, \ldots, m$. Hence $R / M_{i} \cong \mathbf{Z} / 2 \mathbf{Z}$ for $i=1, \ldots, m$. Thus, $R \cong R / M_{1} \times \cdots \times R / M_{m} \cong F_{1} \times \cdots \times F_{m}$ as rings, where $F_{i}=\mathbf{Z} / 2 \mathbf{Z}$ for $i=1, \ldots, m$.

Conversely, suppose that $R \cong T=F_{1} \times \cdots \times F_{m}$ as rings for some $m \geq 1$, where $F_{i}=\mathbf{Z} / 2 \mathbf{Z}$ for $i=1, \ldots, m$. It is clear that $t=t^{2}$ for each $t \in T$ and so $Z(T) \cup\{(1+2 \mathbf{Z}, 1+2 \mathbf{Z}, \ldots, 1+2 \mathbf{Z})\}=T$ and so we obtain that $Z(R) \cup\{1\}=R$.

Recall from [3, Exercise 16, p. 111] that a ring $R$ is said to be von Neumann regular if for each $a \in R$ there is an element $b \in R$ such that $a=a^{2} b$. It is clear that any field is von Neumann regular. Moreover, it follows from [3, Exercise 17, p. 111] that if $\left\{F_{\alpha}\right\}_{\alpha \in \Lambda}$ is any collection of fields, then $\prod_{\alpha \in \Lambda} F_{\alpha}$ is von Neumann regular.

Remark 2.8 If for a ring $R, Z(R) \cup\{1\}=R$, then $R$ is isomorphic to a subring of a von Neumann regular ring.

Proof Let $\left\{M_{\alpha}\right\}_{\alpha \in \Lambda}$ denote the set of all maximal ideals of $R$. Since $Z(R) \cup\{1\}=R$, it follows that $U(R)=\{1\}$ and hence from Remark 2.5(iii), we obtain that $\cap_{\alpha \in \Lambda} M_{\alpha}=(0)$. Observe that the mapping $\phi: R \rightarrow \prod_{\alpha \in \Lambda} R / M_{\alpha}$ given by $\phi(r)=\left\{r+M_{\alpha}\right\}_{\alpha \in \Lambda}$ is a homomorphism of rings and is injective. Let $T=\prod_{\alpha \in \Lambda} R / M_{\alpha}$. Since $R / M_{\alpha}$ is a field for each $\alpha \in \Lambda$, it follows that $T$ is von Neumann regular. Note that $R \cong \phi(R)$ as rings and $\phi(R)$ is a subring of $T$.

We determine in Proposition 2.11 when a von Neumann regular ring $R$ satisfies $Z(R) \cup\{1\}=R$. We make use of the following lemma in the proof of Proposition 2.11 and some other results of Sect. 4 of this article. For any ideal $I$ of a ring $R$ and an element $r \in R$, we denote $r+I$, the residue class of $r$ modulo $I$ by $\bar{r}$.

Lemma 2.9 Let $I$ be a proper ideal of a ring $R$. Then the multiplicatively closed subset $S=1+I$ of $R$ is saturated if and only if $U(R / I)=\{\overline{1}\}$.

Proof Suppose that the multiplicatively closed subset $S=1+I$ of $R$ is saturated. It is clear that $\{\overline{1}\} \subseteq U(R / I)$. Let $a \in R$ be such that $\bar{a} \in U(R / I)$. Then there exists $b \in R$ such that $a b \in S=1+I$. Since $S$ is saturated, $a, b \in S$. Hence $a=1+i$ for some $i \in I$. Therefore, we obtain that $\bar{a}=\overline{1}$. This proves that $U(R / I) \subseteq\{\overline{1}\}$ and so we obtain that $U(R / I)=\{\overline{1}\}$.

Conversely, suppose that $U(R / I)=\{\overline{1}\}$. Let $x, y \in R$ be such that $x y \in S$. Hence $x y=1+c$ for some $c \in I$. This implies that $\overline{x y}=\overline{1}$ and so $\bar{x}, \bar{y} \in U(R / I)$. Since $U(R / I)=\{\overline{1}\}$, it follows that $\bar{x}=\bar{y}=\overline{1}$. Hence $x, y \in 1+I=S$. This proves that $S=1+I$ is saturated.

We next mention the following corollary to Lemma 2.9, which is also used in the proof of Proposition 2.11.

Corollary 2.10 Let $R$ be a Boolean ring (that is, $r=r^{2}$ for each $r \in R$ ). Then for any ideal $I$ of $R$, the multiplicatively closed subset $S=1+I$ of $R$ is saturated.

Proof If $I=R$, then it is clear that $S=1+I=R$ and hence is saturated. Suppose that $I \neq R$. In view of Lemma 2.9, to prove that the multiplicatively closed subset $S=1+I$ of $R$ is saturated, it is enough to show that $U(R / I)=\{\overline{1}\}$. Note that $R / I$ is also a Boolean ring. We complete the proof of this corollary by showing that for any Boolean ring $T, U(T)=\{1\}$. Let $t \in T$. Suppose that $t \neq 1$. Now from $t=t^{2}$, it follows that $t(1-t)=0$ and hence $t \in Z(T)$. Therefore, $t$ cannot be a unit in $T$. This proves that $U(T)=\{1\}$, and moreover, $Z(T) \cup\{1\}=T$.

The following proposition determines when a von Neumann regular ring $R$ satisfies $Z(R) \cup\{1\}=R$ and also shows that the converse of Corollary 2.10 is true.

Proposition 2.11 Let $R$ be a ring. The following statements are equivalent: 
(i) $R$ is a von Neumann regular ring and $Z(R) \cup\{1\}=R$.

(ii) $R$ is a Boolean ring (that is, $r=r^{2}$ for each $r \in R$ ).

(iii) If $S$ is any subring of $R$, then $Z(S) \cup\{1\}=S$.

(iv) If $I$ is any ideal of $R$, then the multiplicatively closed subset $S=1+I$ of $R$ is saturated.

(v) $R$ is a von Neumann regular ring and $|R / M|=2$ for each maximal ideal $M$ of $R$.

Proof (i) $\Rightarrow$ (ii) Let $r \in R$. Since $R$ is von Neumann regular, we obtain from [3, Exercise 29, p. 113] that $r=u e$ where $u$ is a unit in $R$ and $e$ is an idempotent element of $R$. It now follows from $Z(R) \cup\{1\}=R$ that 1 is the only unit of $R$. Thus, $r=e=e^{2}=r^{2}$.

(ii) $\Rightarrow$ (iii) Let $S$ be any subring of $R$. Then $s=s^{2}$ for each $s \in S$. It is shown in the proof of Corollary 2.10 that for any Boolean ring $T, Z(T) \cup\{1\}=T$. Hence, we obtain that $Z(S) \cup\{1\}=S$.

(iii) $\Rightarrow$ (i) $R$ is a subring of $R$. Hence $Z(R) \cup\{1\}=R$. We next show that $R$ is a von Neumann regular ring. In view of [3, Exercise 16, p. 111], it is enough to prove that $R$ is reduced and $\operatorname{dim} R=0$. Since $U(R)=\{1\}$, we know from Remark 2.5(ii) that $R$ is reduced. Moreover, we obtain from Remark 2.5(i) that $1+1=0$. Hence $R$ contains $S=\{0,1\}$ as a subring. Note that $S \cong \mathbf{Z} / 2 \mathbf{Z}$ as rings. We claim that $R$ is integral over $S$. Let $r \in R$. If $r \in S$, then it is clear that $r$ is integral over $S$. Hence, we may assume that $r \notin S$. Consider the subring $S[r]$ of $R$. It follows from (iii) that $Z(S[r]) \cup\{1\}=S[r]$. Hence $r \in Z(S[r])$ and so there exists $t \in S[r] \backslash\{0\}$ such that $r t=0$. Let $t=s_{0}+s_{1} r+\cdots+s_{n} r^{n}$ for some $n \geq 1, s_{i} \in S$ for $i \in\{0,1 \ldots, n\}$ with $s_{n} \neq 0$. Since $S=\{0,1\}$, it follows that $s_{n}=1$ and from $r t=0$, we obtain that $r^{n+1}+s_{n-1} r^{n}+\cdots+s_{0} r=0$. This proves that $r$ is integral over $S$ and so $R$ is integral over $S$. Now we obtain from [4, Theorem 48] that $\operatorname{dim} R=\operatorname{dim} S=0$.

(i) $\Rightarrow$ (iv) Since (i) $\Rightarrow$ (ii), we obtain that $R$ is a Boolean ring. Hence, we obtain from Corollary 2.10 that for any ideal $I$ of $R$, the multiplicatively closed subset $S=1+I$ of $R$ is saturated.

(iv) $\Rightarrow$ (i) Let $I$ be any ideal of $R$. We claim that $I=\sqrt{I}$. This is clear if $I=R$. Suppose that $I \neq R$. Since the multiplicatively closed subset $S=1+I$ of $R$ is saturated, we obtain from Lemma 2.9 that $U(R / I)=\{\overline{1}\}$. Hence it follows from Remark 2.5(ii) that $R / I$ is a reduced ring. This implies that $I=\sqrt{I}$. Let $r \in R$. Now $R r=\sqrt{R r}=\sqrt{R r^{2}}=R r^{2}$. Hence $r=r^{2} s$ for some $s \in R$. This proves that $R$ is a von Neumann regular ring. As $R$ is a von Neumann regular ring, it is clear that $Z(R) \cup U(R)=R$. Let $S=\{1\}$. Since $S=\bar{S}$ by (iv), it follows that $\{1\}=U(R)$. Hence we obtain that $Z(R) \cup\{1\}=R$. This proves (iv) $\Rightarrow$ (i).

(ii) $\Rightarrow$ (v) Since $R$ is a Boolean ring, $R$ is von Neumann regular. Let $M$ be any maximal ideal of $R$. Since each element of $R$ is idempotent, it follows that each element of the field $R / M$ is idempotent. Hence we obtain that $R / M=\{0+M, 1+M\}$. This proves that $|R / M|=2$ for each maximal ideal $M$ of $R$.

(v) $\Rightarrow$ (ii) Let $r \in R$. Let $M$ be any maximal ideal of $R$. Since $|R / M|=2$, it follows that $r+M=$ $(r+M)^{2}=r^{2}+M$. Hence, $r-r^{2} \in M$ for each maximal ideal $M$ of $R$. As $R$ is a von Neumann regular ring, $R$ is reduced and $\operatorname{dim} R=0$ [3, Exercise 16(d), p. 111]. Thus, $r-r^{2} \in \cap_{P \in \operatorname{Spec}(R)} P=$ the nilradical of $R=(0)$. Hence $r=r^{2}$ for each $r \in R$.

This completes the proof of Proposition 2.11.

The following proposition determines when a multiplicatively closed subset of a ring satisfies $(P)$.

Proposition 2.12 Let $R$ be a ring and let $S$ be a multiplicatively closed subset of $R$. Then $S$ satisfies $(P)$ if and only if $R \backslash S=\cup_{a \in R \backslash S} S a$.

Proof Assume that $S$ satisfies $(P)$. First observe that if $r$ is any element of $R$, then as $1 \in S$, it follows that $r=1 r \in S r$. Hence, we obtain that $R \backslash S \subseteq \cup_{a \in R \backslash S} S a$. Let $a \in R \backslash S$. As $S$ satisfies $(P)$, it is clear that $S a \subseteq R \backslash S$. Hence $\cup_{a \in R \backslash S} S a \subseteq R \backslash S$. Therefore, we obtain that $R \backslash S=\cup_{a \in R \backslash S} S a$.

Conversely, suppose that the multiplicatively closed subset $S$ of $R$ satisfies $R \backslash S=\cup_{a \in R \backslash S} S a$. Let $x, y \in R$ be such that $x, x y \in S$. If $y \notin S$, then as $S y \subseteq R \backslash S$, it follows that $x y \notin S$. This is a contradiction. Hence $y \in S$. This shows that $S$ satisfies $(P)$.

Let $S$ be a multiplicatively closed subset of $R$ with $U(R) \subseteq S$. The following proposition determines when $S$ is saturated.

Proposition 2.13 Let $R$ be a ring and let $S$ be a multiplicatively closed subset of $R$ with $U(R) \subseteq S$. Then $S$ is saturated if and only if $(R \backslash S) \cup\{1\}$ is a multiplicatively closed subset of $R$ and $S$ satisfies $(P)$. 
Proof Suppose that $S$ is saturated. Then it is clear that $(R \backslash S) \cup\{1\}$ is a multiplicatively closed subset of $R$ and $S$ satisfies $(P)$.

Conversely, suppose that $(R \backslash S) \cup\{1\}$ is a multiplicatively closed subset of $R$ and $S$ satisfies $(P)$. Let $a, b \in R$ be such that $a b \in S$. We want to show that $a, b \in S$.

Since $U(R) \subseteq S$ and $S$ is multiplicatively closed, it follows that $a, b \in S$ if either $a$ or $b$ is a unit in $R$. Hence, we may assume that $a \notin U(R)$ and $b \notin U(R)$.

Suppose that $a \notin S$. Since $a b \in S$ and $S$ satisfies $(P)$, it follows that $b \notin S$. Thus $a, b \in(R \backslash S) \cup\{1\}$. By assumption, $(R \backslash S) \cup\{1\}$ is a multiplicatively closed subset of $R$. Hence $a b \in(R \backslash S) \cup\{1\}$. Since $a b \in S$, we obtain that $a b=1$. This is impossible since $a \notin U(R)$. Therefore, $a \in S$. Similarly, it follows that $b \in S$. This proves that $S$ is saturated.

\section{3 (P)-closure of multiplicatively closed sets}

We begin with the following remark.

Remark 3.1 Let $S$ be a multiplicatively closed subset of a ring $R$. Note that if $\mathcal{W}$ is the collection of all multiplicatively closed subsets $T$ of $R$ with the following properties: (i) $S \subseteq T$ and (ii) $T$ satisfies $(P)$, then $S_{1}=\cap_{T \in \mathcal{W}} T \in \mathcal{W}$ and $S_{1}$ is the smallest element of $\mathcal{W}$ with respect to the inclusion relation. That is, $S_{1}$ is the smallest multiplicatively closed subset of $R$ such that $S \subseteq S_{1}$ and $S_{1}$ satisfies $(P)$. We call $S_{1}$, the $(P)$-closure of $S$ and for convenience, we denote it by $(P)-\operatorname{cl}(S)$.

Let $R, S$ be as in Remark 3.1. In the following proposition, we describe the elements of $(P)-\operatorname{cl}(S)$. For any subset $A$ of $R$ and an element $b$ of $R$, we denote the set $\{r \in R \mid r b \in A\}$ by $\left(A:_{R} b\right)$.

Proposition 3.2 Let $S$ be a multiplicatively closed subset of a ring $R$. Then $(P)-\operatorname{cl}(S)=\cup_{s \in S}\left(S:_{R} s\right)$.

Proof For convenience, let us denote the set $\cup_{s \in S}(S: R s)$ by $T$. We first show that $T$ is a multiplicatively closed subset of $R$. Note that since $S$ is a multiplicatively closed subset of $R, S \subseteq\left(S:_{R} S\right)$ for each $s \in S$. Hence we obtain that $S \subseteq T$. As $1 \in S$, it follows that $1 \in T$. Let $t_{1}, t_{2} \in T$. Now there exist $s_{1}, s_{2} \in S$ such that $t_{1} s_{1} \in S$ and $t_{2} s_{2} \in S$. Since $S$ is a multiplicatively closed subset of $R$, it follows that $s_{1} s_{2} \in S$ and $t_{1} s_{1} t_{2} s_{2} \in S$. Hence, we obtain that $t_{1} t_{2} \in\left(S:_{R} s_{1} s_{2}\right) \subseteq T$. This proves that $T$ is a multiplicatively closed subset of $R$.

We next verify that $T$ satisfies $(P)$. Let $r_{1}, r_{2} \in R$ be such that $r_{1} r_{2}, r_{1} \in T$. Now there exist $s_{1}, s \in S$ such that $r_{1} s_{1} \in S$ and $r_{1} r_{2} s \in S$. Observe that $r_{1} s_{1} s \in S$ and $r_{2} \in\left(S: R r_{1} s_{1} s\right) \subseteq T$. This shows that $T$ satisfies $(P)$.

Let $T_{1}$ be any multiplicatively closed subset of $R$ such that $S \subseteq T_{1}$ and $T_{1}$ satisfies $(P)$. We assert that $T \subseteq T_{1}$. Let $t \in T$. Then $t s \in S$ for some $s \in S$. Now $t s, s \in S \subseteq T_{1}$ and as $T_{1}$ satisfies $(P)$, it follows that $t \in T_{1}$. Hence $T \subseteq T_{1}$.

This proves that $T$ is the $(P)$-closure of $S$. Thus,

$(P)-\operatorname{cl}(S)=\cup_{s \in S}\left(S:_{R} S\right)$.

We next provide some examples to illustrate Proposition 3.2. We also state and prove some corollaries to Proposition 3.2.

Example 3.3 Let $R$ be an integral domain which is not a field. Let $I$ be a nonzero proper ideal of $R$. Let $S=(I \backslash\{0\}) \cup\{1\}$. It is noted in Remark 2.4 that $S$ is a multiplicatively closed subset of $R$ and $S$ does not satisfy $(P)$. We assert that $(P)-\operatorname{cl}(S)=R \backslash\{0\}$. The above assertion can be verified directly. However, to illustrate Proposition 3.2, we verify it using Proposition 3.2. We know from Proposition 3.2 that $(P)-\operatorname{cl}(S)=$ $\cup_{s \in S}(S: R s)$. Since $S \subseteq R \backslash\{0\}$ and $R$ is an integral domain, it follows that $\left(S:_{R} s\right) \subseteq R \backslash\{0\}$ for any $s \in S$. Hence we obtain that $(\bar{P})-\operatorname{cl}(S) \subseteq R \backslash\{0\}$. Let $a \in I \backslash\{0\}$. Now for any $r \in R \backslash\{0\}, r a \in I \backslash\{0\} \subseteq S$. Thus, $r \in(S: R a) \subseteq(P)-\operatorname{cl}(S)$. This proves that $R \backslash\{0\} \subseteq(P)-\operatorname{cl}(S)$ and hence $(P)-\operatorname{cl}(S)=R \backslash\{0\} . \quad \square$

It follows from the above paragraph that if $n$ is any natural number with $n>1$, then the multiplicatively closed subset $S=(n \mathbf{Z} \backslash\{0\}) \cup\{1\}$ of $\mathbf{Z}$ is such that $(P)-\operatorname{cl}(S)=\mathbf{Z} \backslash\{0\}$.

Let $T$ be a commutative ring with identity and let $R$ be a subring of $T$. Let $S$ be a multiplicatively closed subset of $R$. Observe that $S$ can also be regarded as a multiplicatively closed subset of $T$. We call the smallest multiplicatively closed subset of $T$ which contains $S$ and satisfies $(P)$, the $(P)$-closure of $S$ in $T$ and for convenience, we denote the $(P)$-closure of $S$ in $T$ by $(P)-\operatorname{cl}_{T}(S)$. Thus, $(P)-\operatorname{cl}(S)=(P)-\mathrm{cl}_{R}(S)$. In Corollary 3.4, we determine $(P)-\operatorname{cl}_{T}(S)$, under the hypothesis that $T$ is an integral domain and $S=R \backslash\{0\}$. 
Corollary 3.4 Let $R$ be a subring of an integral domain $T$. Let $K$ be the quotient field of $R$. Let $S=R \backslash\{0\}$. Then the following hold:

(a) $S=R \backslash\{0\}$ is a multiplicatively closed subset of $T$ and $(P)-\operatorname{cl}_{T}(S)=K \cap(T \backslash\{0\})$.

(b) The following statements are equivalent:

(i) $S=R \backslash\{0\}$ regarded as a multiplicatively closed subset of $T$ satisfies $(P)$.

(ii) $K \cap(T \backslash\{0\})=S$.

(iii) If $S_{1} \subseteq R \backslash\{0\}$ is any multiplicatively closed subset of $R$ and if $S_{1}$ satisfies $(P)$, then $S_{1}$ regarded as a multiplicatively closed subset of $T$ satisfies $(P)$.

Proof (a) Since $R$ is an integral domain, $S=R \backslash\{0\}$ is a multiplicatively closed subset of $R$ (indeed, $S$ is a saturated multiplicatively closed subset of $R$ ). As $T$ contains $R$ as a subring, it is clear that $S$ is a multiplicatively closed subset of $T$. We now verify that $(P)-\operatorname{cl}_{T}(S)=K \cap(T \backslash\{0\})$. We know from Proposition 3.2 that $(P)-\operatorname{cl}_{T}(S)=\cup_{s \in S}\left(S:_{T} S\right)$. Let $t \in(P)-\operatorname{cl}_{T}(S)$. Then there exists $s \in S=R \backslash\{0\}$ such that $s t \in S$. Hence $t \neq 0$ and $t=s t / s \in K \cap(T \backslash\{0\})$. Thus, $(P)-\operatorname{cl}_{T}(S) \subseteq K \cap(T \backslash\{0\})$. Let $t \in K \cap(T \backslash\{0\})$. Then $t=a / b$ for some $a, b \in R \backslash\{0\}=S$. Hence $t b=a \in S$. This shows that $t \in\left(S:_{T} b\right) \subseteq(P)-\operatorname{cl}_{T}(S)$. This proves that $(P)-\operatorname{cl}_{T}(S)=K \cap(T \backslash\{0\})$.

(b) (i) $\Leftrightarrow$ (ii) $S$ regarded as a multiplicatively closed subset of $T$ satisfies $(P)$ if and only if $(P)-\operatorname{cl}_{T}(S)=$ $S$. Hence it follows from (a) that $S$ regarded as a multiplicatively closed subset of $T$ satisfies $(P)$ if and only if $K \cap(T \backslash\{0\})=S$.

(ii) $\Rightarrow$ (iii) Let $t_{1}, t_{2} \in T$ be such that $t_{1} t_{2}, t_{1} \in S_{1}$. Hence $t_{2}=t_{1} t_{2} / t_{1} \in K \cap(T \backslash\{0\})=R \backslash\{0\}$ by (ii). Thus, $t_{1}, t_{2} \in R$ and are such that $t_{1} t_{2}, t_{1} \in S_{1}$. Since $S_{1}$ regarded as a multiplicatively closed subset of $R$ satisfies $(P)$, we obtain that $t_{2} \in S_{1}$. This proves that $S_{1}$ regarded as a multiplicatively closed subset of $T$ satisfies $(P)$.

(iii) $\Rightarrow$ (i) Since $R$ is an integral domain, $S=R \backslash\{0\}$ is a saturated multiplicatively closed subset of $R$. Hence $S$ satisfies $(P)$. Now we obtain from (iii) that $S$ regarded as a multiplicatively closed subset of $T$ satisfies $(P)$.

We next provide the following remark which illustrates Corollary 3.4.

Remark 3.5 (i) Let $R$ be a subring of an integral domain $T$. Suppose that $R$ is integrally closed and $T$ is integral over $R$. Let $S_{1} \subseteq R \backslash\{0\}$ be a multiplicatively closed subset of $R$ and $S_{1}$ satisfies $(P)$. Then $S_{1}$ regarded as a multiplicatively closed subset of $T$ satisfies $(P)$.

(ii) Let $K$ be any algebraic number field. Let $R_{K}$ denote the ring of algebraic integers in $K$. That is, $R_{K}=$ $\{\alpha \in K \mid \alpha$ is integral over $\mathbf{Z}\}$. Let $S_{1} \subseteq \mathbf{Z} \backslash\{0\}$ be a multiplicatively closed subset of $\mathbf{Z}$ and suppose that $S_{1}$ satisfies $(P)$. Then $S_{1}$ regarded as a multiplicatively closed subset of $R_{K}$ satisfies $(P)$.

(iii) Let $T$ be an integral domain. Let $L$ denote the quotient field of $T$. Let $R$ be a subring of $T$ and let $K$ be its quotient field. Let $S=R \backslash\{0\}$. If $L$ is algebraic over $K$, then the saturation of $S$ regarded as a multiplicatively closed subset of $T$ equals $T \backslash\{0\}$. Thus $S$ regarded as a multiplicatively closed subset of $T$ is saturated if and only if $T=R$.

(iv) Let $F[x]$ denote the polynomial ring in one variable over a field $F$. Let $R=F\left[x^{2}, x^{3}\right]$ and let $T=$ $F[x, y]$, the polynomial ring in two variables over $F$. Let $S=R \backslash\{0\}$. Then $(P)-\operatorname{cl}_{T}(S)=F[x] \backslash\{0\}$

Proof (i) Let $S_{1} \subseteq R \backslash\{0\}$ be a multiplicatively closed subset of $R$ and suppose that $S_{1}$ satisfies $(P)$. We assert that $S_{1}$ regarded as a multiplicatively closed subset of $T$ satisfies $(P)$. To prove this assertion, in view of Corollary 3.4(b) (ii) $\Rightarrow$ (iii), it is enough to show that $K \cap(T \backslash\{0\})=R \backslash\{0\}$, where $K$ is the quotient field of $R$. It is clear that $R \backslash\{0\} \subseteq K \cap(T \backslash\{0\})$. Let $t \in K \cap(T \backslash\{0\})$. Now $t \in K$ is integral over $R$. Since $R$ is integrally closed, we obtain that $t \in R$. Hence, $K \cap(T \backslash\{0\}) \subseteq R \backslash\{0\}$ and so $K \cap(T \backslash\{0\})=R \backslash\{0\}$. Hence, $S_{1}$ regarded as a multiplicatively closed subset of $T$ satisfies $(P)$.

(ii) This follows immediately from (i) since $R_{K}$ is integral over $\mathbf{Z}$ and $\mathbf{Z}$ is integrally closed.

(iii) We know from [1, Exercise 7(ii), p. 44] that the saturation of $S$ regarded as a multiplicatively closed subset of $T$ equals $T \backslash\left(\cup_{\alpha \in \Lambda} Q_{\alpha}\right)$, where $\left\{Q_{\alpha}\right\}_{\alpha \in \Lambda}$ is the collection of prime ideals of $T$ which do not meet $S$. Thus, for each $\alpha \in \Lambda, Q_{\alpha} \cap R=(0)$. Since $T$ is algebraic over $R, A \cap R \neq(0)$ for each nonzero ideal $A$ of $T$. Hence $Q_{\alpha}=(0)$ for each $\alpha \in \Lambda$. Therefore, we obtain that the saturation of $S$ regarded as a multiplicatively closed subset of $T$ equals $T \backslash\{0\}$.

Now, $S=R \backslash\{0\}$ regarded as a multiplicatively closed subset of $T$ is saturated if and only if $S=$ the saturation of $S$ regarded as a multiplicatively closed subset of $T=T \backslash\{0\}$ if and only if $T=R$. 
(iv) Since $F(x)$ is the quotient field of $R=F\left[x^{2}, x^{3}\right]$, it follows from Corollary 3.4(a) that $(P)-\mathrm{cl}_{T}(S)=$ $F(x) \cap(T \backslash\{0\})$. It is easy to show that $F(x) \cap(F[x, y] \backslash\{0\})=F[x] \backslash\{0\}$. Hence we obtain that $(P)-\operatorname{cl}_{T}(S)=F[x] \backslash\{0\}$.

We next have the following remark.

Remark 3.6 Let $R$ be a subring of an integral domain $T$. Let $a \in R \backslash\{0\}$ be such that $a$ is not a unit in $R$. Let $S=\left\{a^{n} \mid n \geq 0\right\}$. Then the following hold:

(i) $(P)-\operatorname{cl}_{T}(S)=S$ if $a \notin U(T)$.

(ii) $(P)-\operatorname{cl}_{T}(S)=\left\{a^{n} \mid n \in \mathbf{Z}\right\}$ if $a \in U(T)$.

Proof (i) If $a$ is not a unit in $T$, then it follows from Example 2.1(ii) that $S$ satisfies $(P)$ when regarded as a multiplicatively closed subset of $T$. Hence we obtain that $(P)-\mathrm{cl}_{T}(S)=S$.

(ii) We know from Proposition 3.2 that $(P)-\mathrm{cl}_{T}(S)=\cup_{s \in S}\left(S:_{T} S\right)$. Suppose that $a$ is a unit in $T$. Let $n \in \mathbf{Z}$. If $n \geq 0$, then $a^{n} \in S$ and so $a^{n} \in\left(S:_{T}: s\right)$ for each $s \in S$. If $n<0$, then $a^{-n} \in S$ and $a^{n} a^{-n}=1 \in S$. Thus $a^{n} \in\left(S: T a^{-n}\right)$. This proves that $\left\{a^{n} \mid n \in \mathbf{Z}\right\} \subseteq(P)-\operatorname{cl}_{T}(S)$. Let $t \in(P)-\operatorname{cl}_{T}(S)$. Then $t a^{m} \in S=\left\{a^{k} \mid k \geq 0\right\}$ for some $m \geq 0$. Hence $t a^{m}=a^{k}$ for some $k \geq 0$. This implies that $t=a^{k-m} \in\left\{a^{n} \mid n \in \overline{\mathbf{Z}}\right\}$. Thus $(P)-\operatorname{cl}_{T}(\bar{S}) \subseteq\left\{a^{n} \mid n \in \mathbf{Z}\right\}$. This shows that $(P)-\mathrm{cl}_{T}(S)=\left\{a^{n} \mid n \in \mathbf{Z}\right\}$.

Let $R$ be a subring of a ring $T$. In the following remark, we provide some sufficient condition under which the following holds: if $S_{1} \subseteq R \backslash\{0\}$ is any multiplicatively closed subset of $R$ and if $S_{1}$ satisfies $(P)$, then $S_{1}$ satisfies $(P)$ when regarded as a multiplicatively closed subset of $T$.

Remark 3.7 (i) Let $R$ be a subring of an integral domain $T$. Suppose that for each $r \in R, r T \cap R=r R$. Let $S_{1} \subseteq R \backslash\{0\}$ be a multiplicatively closed subset of $R$. If $S_{1}$ satisfies $(P)$, then $S_{1}$ satisfies $(P)$ when regarded as a multiplicatively closed subset of $T$.

(ii) Let $R$ be an integral domain. Let $T=R[x]$ (resp. $T=R[[x]]$ ). If $S_{1} \subseteq R \backslash\{0\}$ is any multiplicatively closed subset of $R$ which satisfies $(P)$, then $S_{1}$ satisfies $(P)$ when regarded as a multiplicatively closed subset of $T$.

Proof (i) Let $K$ denote the quotient field of $R$. Let $S_{1} \subseteq R \backslash\{0\}$ be any multiplicatively closed subset of $R$ and suppose that $S_{1}$ satisfies $(P)$. To prove that $S_{1}$ regarded as a multiplicatively closed subset of $T$ satisfies $(P)$, in view of Corollary 3.4(b) (ii) $\Rightarrow$ (iii), it is enough to show that $K \cap(T \backslash\{0\})=R \backslash\{0\}$. It is clear that $R \backslash\{0\} \subseteq K \cap(T \backslash\{0\})$. Let $t \in K \cap(T \backslash\{0\})$. Note that $t=a / b$ for some $a, b \in R \backslash\{0\}$. This implies that $a=b t \in b T \cap R$. By hypothesis, $b T \cap R=b R$. Hence we obtain that $t=a / b \in R$. This shows that $K \cap(T \backslash\{0\}) \subseteq R \backslash\{0\}$ and so $K \cap(T \backslash\{0\})=R \backslash\{0\}$. Hence we obtain that $S_{1}$ regarded as a multiplicatively closed subset of $T$ satisfies $(P)$.

If $R$ is a subring of a ring $T$ and if $T$ regarded as an $R$-module is faithfully flat, then it is known that for any ideal $I$ of $R, I T \cap R=I$ [5, Theorem 7.5(ii)].

(ii) Let $S_{1} \subseteq R \backslash\{0\}$ be any multiplicatively closed subset of $R$ and suppose that $S_{1}$ satisfies $(P)$. Let $T=R[x]$ (resp. $T=R[[x]])$. Since for any $r \in R, r T \cap R=r R$, it follows from (i) that $S_{1}$ regarded as a multiplicatively closed subset of $T$ satisfies $(P)$.

In Example 3.9, we illustrate that Remark 3.7(ii) may fail to hold if $R$ is not an integral domain. We make use of the following lemma in the verification of Example 3.9.

Lemma 3.8 Let $R$ be a subring of a ring $T$. Let $I$ be an ideal of $R$. Let $S_{1}=1+I$. If $\left((0):_{R} I\right)+I=R$, then $(P)-\operatorname{cl}_{T}\left(S_{1}\right)=1+I T$.

Proof Since $I T$ is an ideal of $T$, it follows from Example 2.1(i) that the multiplicatively closed subset $1+I T$ of $T$ satisfies $(P)$. As $S_{1}=1+I \subseteq 1+I T$, we obtain that $(P)-\mathrm{cl}_{T}\left(S_{1}\right) \subseteq 1+I T$. We next verify that $1+I T \subseteq(P)-\operatorname{cl}_{T}\left(S_{1}\right)$. By hypothesis, $\left((0):_{R} I\right)+I=R$. Hence there exist $b \in\left((0):_{R} I\right), a \in I$ such that $a+b=1$. Thus $b=1-a \in S_{1} \cap((0): R I)$. Let $t \in 1+I T$. Note that $t=1+y$ for some $y \in I T$. As $b \in\left((0):_{R} I\right)$ and $y \in I T$, it follows that $b y=0$. Hence $t b=(1+y) b=b \in S_{1}$. This shows that $t \in\left(S_{1}:_{T} b\right)$. It follows from Proposition 3.2 that $\left(S_{1}: T b\right) \subseteq(P)-\operatorname{cl}_{T}\left(S_{1}\right)$. Hence we obtain that $t \in(P)-\operatorname{cl}_{T}\left(S_{1}\right)$. This proves that $1+I T \subseteq(P)-\operatorname{cl}_{T}\left(S_{1}\right)$ and so we obtain that $(P)-\mathrm{cl}_{T}\left(S_{1}\right)=1+I T$. 
Example 3.9 Let $R=\mathbf{Z} / 6 \mathbf{Z}$. Let $T=R[x]$ be the polynomial ring in one variable over $R$. Let $S_{1}=\overline{1}+\overline{2} R$. Then the following statements hold:

(i) $S_{1}$ is a multiplicatively closed subset of $R$ and $S_{1}$ satisfies $(P)$.

(ii) $(P)-\operatorname{cl}_{T}\left(S_{1}\right)=\overline{1}+\overline{2} T$. Hence $S_{1}$ regarded as a multiplicatively closed subset of $T$ does not satisfy $(P)$.

\section{Proof (i) This is obvious.}

(ii) Let $I=\overline{2} R$. As the product of $\overline{2}$ and $\overline{3}$ is $\overline{0}$, it follows that $\overline{3} \in((\overline{0}): R I)$. Since $\overline{2}+\overline{3}=\overline{5} \in U(R)$, it follows that $I+\left((\overline{0}):_{R} I\right)=R$. Now on applying Lemma 3.8 with $T=R[x]$, we obtain that $(P)-\operatorname{cl}_{T}\left(S_{1}\right)=\overline{1}+\overline{2} T$. Note that $\overline{1}+\overline{2} x \in(P)-\operatorname{cl}_{T}\left(S_{1}\right)$ but $\overline{1}+\overline{2} x \notin S_{1}$. Hence $(P)-\operatorname{cl}_{T}\left(S_{1}\right) \neq S_{1}$. Therefore, $S_{1}$ regarded as a multiplicatively closed subset of $T$ does not satisfy $(P)$.

\section{Some results on saturated multiplicatively closed sets of the form $1+I$}

Throughout Sects. 4 and 5, we use $\mathcal{R}$ to denote the collection of all commutative rings $R$ with identity satisfying the property that $R$ admits only a finite number of saturated multiplicatively closed sets of the form $1+I$ where $I$ is an ideal of $R$. If $R$ is semiquasilocal, then it is shown in Proposition 4.3(i) that $R \in \mathcal{R}$. Let $R$ be an integral domain, $\operatorname{dim} R=1$ and if $R$ has Noetherian spectrum, then we prove in Proposition 4.5 that $R \in \mathcal{R}$. Moreover, we mention some examples to illustrate the results proved in this section. We begin with the following proposition.

Proposition 4.1 Let I be an ideal of $\mathbf{Z}$. Then the multiplicatively closed subset $S=1+I$ of $\mathbf{Z}$ is saturated if and only if either $I=\mathbf{Z}$ or $I=2 \mathbf{Z}$.

Proof Let $I$ be an ideal of $\mathbf{Z}$. Suppose that the multiplicatively closed subset $S=1+I$ of $\mathbf{Z}$ is saturated and $I \neq \mathbf{Z}$. If $I=(0)$, then $S=\{1\}$. As $(-1)^{2}=1 \in S$, whereas $-1 \notin S$, it follows that $I \neq(0)$. Note that $I=n \mathbf{Z}$ for some $n \geq 2$. It is well known that $|U(\mathbf{Z} / n \mathbf{Z})|=\phi(n)$, where $\phi$ is the Euler's phi-function. Since $S$ is saturated, it follows from Lemma 2.9 that $|U(\mathbf{Z} / n \mathbf{Z})|=1$. Thus $\phi(n)=1$. As $\phi(n) \geq 2$ for all $n \geq 3$, we obtain that $n=2$. Hence either $I=\mathbf{Z}$ or $I=2 \mathbf{Z}$.

Conversely, suppose that either $I=\mathbf{Z}$ or $I=2 \mathbf{Z}$. If $I=\mathbf{Z}$, then $S=\mathbf{Z}$ is clearly saturated. If $I=2 \mathbf{Z}$, then as $U(\mathbf{Z} / 2 \mathbf{Z})=\{\overline{1}\}$, it follows from Lemma 2.9 that $S=1+2 \mathbf{Z}$ is saturated (this also follows from the fact that if the product of two integers is odd, then both of them must be odd).

We make use of the following fact in the proof of Proposition 4.3.

Fact 4.2 Let I be a proper ideal of a ring $R$. If the multiplicatively closed subset $S=1+I$ of $R$ is saturated, then $I=\cap_{M \in \mathcal{C}} M$, where $\mathcal{C}=\{M \mid M$ is a maximal ideal of $R$ with $M \supseteq I\}$.

Proof Let $I$ be a proper ideal of $R$ such that the multiplicatively closed subset $S=1+I$ of $R$ is saturated. We know from Lemma 2.9 that $U(R / I)=\{\overline{1}\}$. Hence it follows from Remark 2.5(iii) that the Jacobson radical of $R / I$ is the zero ideal of $R / I$. Let $\mathcal{C}=\{M \mid M$ is a maximal ideal of $R$ with $M \supseteq I\}$. Thus $(0+I)=J(R / I)=$ $\cap_{M \in \mathcal{C}} M / I$. Hence it follows that $I=\cap_{M \in \mathcal{C}} M$.

Let $R$ be a semiquasilocal ring (that is, $R$ has only a finite number of maximal ideals). Proposition 4.3 determines ideals $I$ of $R$ such that the multiplicatively closed subset $S=1+I$ of $R$ is saturated.

Proposition 4.3 Let $R$ be a semiquasilocal ring and let $\left\{M_{1}, \ldots, M_{n}\right\}$ denote the set of all maximal ideals of $R$. Then the following hold:

(i) $R \in \mathcal{R}$.

(ii) $S=\{1\}$ is saturated if and only if $R \cong F_{1} \times \cdots \times F_{n}$ as rings, where $F_{i}=\mathbf{Z} / 2 \mathbf{Z}$ for $i=1, \ldots, n$.

(iii) Let $\mathcal{C}=\{M \mid M$ is a maximal ideal of $R$ with $M \supseteq I\}$, where $I$ is an ideal of $R$ with $I \neq R$. Let $m$ be the number of elements in $\mathcal{C}$. Then $S=1+I$ is saturated if and only if $R / I \cong F_{1} \times \cdots \times F_{m}$ as rings, where $F_{i}=\mathbf{Z} / 2 \mathbf{Z}$ for $i=1, \ldots, m$.

Proof (i) Let $I$ be any proper ideal of $R$ such that the multiplicatively closed subset $S=1+I$ of $R$ is saturated. Let $\mathcal{C}$ be the collection of all maximal ideals $M$ of $R$ such that $M \supseteq I$. We know from Fact 4.2 that $I=\cap_{M \in \mathcal{C}} M$. Since $R$ has only a finite number of maximal ideals, it follows that there can be only a finite number of proper ideals $I$ of $R$ such that $S=1+I$ is saturated. Hence we obtain that $R \in \mathcal{R}$. 
(ii) We know from Lemma 2.9 that the multiplicatively closed subset $S=\{1\}$ of $R$ is saturated if and only if $U(R)=\{1\}$. Thus if $R \cong F_{1} \times \cdots \times F_{n}$ as rings, where $F_{i}=\mathbf{Z} / 2 \mathbf{Z}$ for $i=1, \ldots, n$, then it is clear that $U(R)=\{1\}$ and so $S=\{1\}$ is saturated.

Suppose that $S=\{1\}$ is saturated. Then $U(R)=\{1\}$. Moreover, we know from Fact 4.2 that $J(R)=$ $\cap_{i=1}^{n} M_{i}=(0)$. Using the facts that $U(R)=\{1\}$ and $\cap_{i=1}^{n} M_{i}=(0)$, it follows as in the proof of Proposition 2.7 that $R \cong F_{1} \times \cdots \times F_{n}$ as rings, where $F_{i}=\mathbf{Z} / 2 \mathbf{Z}$ for $i=1, \ldots, n$.

(iii) Let $I$ be an ideal of $R$ with $I \neq R$. Observe that the multiplicatively closed subset $S=1+I$ of $R$ is saturated if and only if $\{\overline{1}\}$ is a saturated multiplicatively closed subset of the ring $R / I$. If $\mathcal{C}$ is the collection of all maximal ideals $M$ of $R$ with $M \supseteq I$, then $\{M / I \mid M \in \mathcal{C}\}$ is the set of all maximal ideals of $R / I$. By assumption $\mathcal{C}$ contains exactly $m$ elements. Hence $R / I$ is a semiquasilocal ring with exactly $m$ maximal ideals. Therefore, we obtain from the above discussion and ( $i i$ ) that $S=1+I$ is saturated if and only if $\{\overline{1}\}$ is a saturated multiplicatively closed subset of $R / I$ if and only if $R / I \cong F_{1} \times \cdots \times F_{m}$ as rings, where $F_{i}=\mathbf{Z} / 2 \mathbf{Z}$ for $i=1, \ldots, m$.

Let $R$ be an integral domain. If $R$ has Noetherian spectrum and if $\operatorname{dim} R=1$, then we prove in Proposition 4.5 that $R \in \mathcal{R}$. First it is useful to recall the following.

Recall from [6] that a ring $R$ has Noetherian spectrum, if the topological space $\operatorname{Spec}(R)$ (that is, the set of all prime ideals of $R$ endowed with the Zariski topology) is Noetherian. It is well known that a ring $R$ has Noetherian spectrum if and only if $R$ satisfies ascending chain condition on radical ideals of $R$ [6]. Moreover, a ring $R$ has Noetherian spectrum if and only if the following conditions hold: (i) $R$ satisfies ascending chain condition on prime ideals of $R$, (ii) each proper ideal of $R$ admits only a finite number of prime ideals minimal over it [4, Theorem 88 and Exercise 25, p. 65].

We make use of the following lemma in the proof of Proposition 4.5.

Lemma 4.4 Let $R$ be an integral domain. Suppose that $R$ has Noetherian spectrum and $\operatorname{dim} R=1$. Let $\mathcal{M}$ denote the collection of all maximal ideals $M$ of $R$ such that the multiplicatively closed subset $S=1+M$ of $R$ is saturated. Then the collection $\mathcal{M}$ is finite.

Proof Suppose that $\mathcal{M}$ is infinite. Then there exist distinct maximal ideals $M_{1}, M_{2}, M_{3}, \ldots$ of $R$ such that $M_{n} \in \mathcal{M}$ for each $n \in \mathbf{N}$. Since the multiplicatively closed subset $S_{n}=1+M_{n}$ of $R$ is saturated for each $n \in \mathbf{N}$, it follows from Lemma 2.9 that $U\left(R / M_{n}\right)=\{\overline{1}\}$. As $R / M_{n}$ is a field for each $n \in \mathbf{N}$, we obtain that $R / M_{n}=\{\overline{0}, \overline{1}\}$.

Let $a$ be a nonzero nonunit of $R$. Since $R$ has Noetherian spectrum, the ideal $R a$ has only a finite number of prime ideals minimal over it. As $R$ is an integral domain and $\operatorname{dim} R=1$, it follows that $a$ can belong to only a finite number of maximal ideals of $R$. Hence, there exists $m \in \mathbf{N}$ such that $a \notin M_{n}$ for all $n \geq m$. Therefore, we obtain that $a+M_{n}=1+M_{n}$ for all $n \geq m$. Thus $a-1 \in \cap_{n \geq m} M_{n}$. Since any nonzero element of $R$ can belong to only a finite number of maximal ideals, it follows that $a-1=0$. Hence $a=1$. This is in contradiction to the choice of $a$.

This proves that $\mathcal{M}$ is finite.

We next have the following proposition the proof of which is a consequence of Fact 4.2 and Lemma 4.4.

Proposition 4.5 Let $R$ be an integral domain. Suppose that $R$ has Noetherian spectrum and $\operatorname{dim} R=1$. Then $R \in \mathcal{R}$.

Proof Let $\mathcal{C}$ denote the collection of all ideals $I$ of $R$ such that the multiplicatively closed subset $1+I$ of $R$ is saturated. We know from Lemma 4.4 that $\mathcal{C}$ can contain only a finite number of maximal ideals of $R$. Let $I$ be a nonzero proper ideal of $R$ such that $I \in \mathcal{C}$. Let $\mathcal{W}$ be the collection of all maximal ideals $M$ of $R$ such that $M \supseteq I$. Since $R$ is a one-dimensional integral domain and has Noetherian spectrum, it follows that $\mathcal{W}$ is finite. Let $\mathcal{W}=\left\{M_{1}, \ldots, M_{n}\right\}$. We know from Fact 4.2 that $I=\cap_{M \in \mathcal{W}} M=\cap_{i=1}^{n} M_{i}$. Now, we obtain from the Chinese remainder theorem [1, Proposition 1.10(ii)] that $R / I \cong R / M_{1} \times \cdots \times R / M_{n}$ as rings. Hence, $U(R / I) \cong U\left(R / M_{1} \times \cdots \times R / M_{n}\right)=U\left(R / M_{1}\right) \times \cdots \times U\left(R / M_{n}\right)$ as groups. As $U(R / I)$ is trivial by Lemma 2.9 , we obtain that $U\left(R / M_{i}\right)$ is trivial for $i=1, \ldots, n$. Hence by Lemma 2.9, we obtain that $M_{i} \in \mathcal{C}$ for $i=1, \ldots, n$. Since $\mathcal{C}$ contains only a finite number of maximal ideals, we obtain from the above discussion that the collection $\mathcal{C}$ is finite. This shows that $R$ can admit only a finite number of ideals $I$ such that the multiplicatively closed subset $S=1+I$ of $R$ is saturated, and hence we obtain that $R \in \mathcal{R}$.

We next mention an example to illustrate that in Proposition 4.5, the hypothesis that $R$ has Noetherian spectrum cannot be omitted. 
Example 4.6 The example mentioned here is a slight modification of [3, Example 42.6]. Let $\left\{p_{1}=2, p_{2}=\right.$ $3, \ldots\}$ be the set of all positive primes of $\mathbf{Z}$. Note that by [3, Theorem 42.5], there exists an algebraic integer $t_{1}$ with $\left[\mathbf{Q}\left(t_{1}\right): \mathbf{Q}\right]=2$ such that $2 \mathbf{Z}=p_{1} \mathbf{Z}$ decomposes with respect to $\mathbf{Q}\left(t_{1}\right)$. That is, if $D_{1}$ is the integral closure of $\mathbf{Z}$ in $\mathbf{Q}\left(t_{1}\right)$, then there exist distinct prime ideals $M_{1}^{(1)}, M_{2}^{(1)}$ of $D_{1}$ such that $2 D_{1}=M_{1}^{(1)} M_{2}^{(1)}$. Now as any nonzero prime ideal of $D_{1}$ is a maximal ideal, it is clear that $M_{j}^{(1)}(j=1,2)$ are the only prime ideals of $D_{1}$ which are lying over $2 \mathbf{Z}$ in $\mathbf{Z}$ and moreover, it follows from [3, Remark 41.9] that $D_{1} / M_{j}^{(1)} \cong \mathbf{Z} / 2 \mathbf{Z}$ as fields for $j=1,2$ (1). Note that in view of [3, Remark 41.9] there can exist at most two prime ideals of $D_{1}$ which are lying over $p_{2} \mathbf{Z}$. Now it follows from [3, Theorem 42.5] that there exists an algebraic integer $t_{2}$ such that $\left[F_{1}\left(t_{2}\right): F_{1}\right]=2$, where $F_{1}=\mathbf{Q}\left(t_{1}\right)$ with the following properties: each prime ideal of $D_{1}$ which lies over $p_{2} \mathbf{Z}$ in $\mathbf{Z}$ remains inertial with respect to $F_{2}$ and both the prime ideals $M_{1}^{(1)}$ and $M_{2}^{(1)}$ decompose with respect to $F_{2}$. If $D_{2}$ denotes the integral closure of $\mathbf{Z}$ in $F_{2}$, then there exist prime ideals $M_{j}^{(2)}$ of $D_{2}$ for $j=1,2,3,4$ such that $M_{1}^{(1)} D_{2}=M_{1}^{(2)} M_{2}^{(2)}$ and $M_{2}^{(1)} D_{2}=M_{3}^{(2)} M_{4}^{(2)}$. Observe that there are exactly four prime ideals of $D_{2}$ which are lying over $2 \mathbf{Z}$ in $\mathbf{Z}$ and the set of such prime ideals is precisely $\left\{M_{j}^{(2)} \mid j \in\{1,2,3,4\}\right.$. Moreover, it follows from [3, Remark 41.9] that $D_{2} / M_{j}^{(2)} \cong D_{1} / M_{1}^{(1)}$ for $j=1,2$ and $D_{2} / M_{j}^{(2)} \cong D_{1} / M_{2}^{(1)}$ for $j=3$, 4. Hence, we obtain from (1) that $D_{2} / M_{j}^{(2)} \cong \mathbf{Z} / 2 \mathbf{Z}$ as fields for $j=1,2,3,4$.

Assume by induction that we have chosen algebraic integers $t_{1}, t_{2}, \ldots, t_{k}$ such that the following conditions are satisfied:

(a) $\left[F_{i+1}: F_{i}\right]=2$ for each $i \in\{1, \ldots, k-1\}$, where $F_{i}=\mathbf{Q}\left(t_{1}, \ldots, t_{i}\right)$.

(b) Let $i \in\{1, \ldots, k\}$ and let $D_{i}$ denote the integral closure of $\mathbf{Z}$ in $F_{i}$. With this notation, there are exactly $2^{i}$ prime ideals $\left\{M_{j}^{(i)}\right\}_{j=1}^{2^{i}}$ of $D_{i}$ which are lying over $p_{1} \mathbf{Z}=2 \mathbf{Z}$ in $\mathbf{Z}$ for $i=1, \ldots, k$. Moreover, $D_{i} / M_{j}^{(i)} \cong \mathbf{Z} / 2 \mathbf{Z}$ as fields for $j=1, \ldots, 2^{i}$. Furthermore, each of the prime ideals $M_{j}^{(i)}$ for $j=1, \ldots, 2^{i}$ decomposes with respect to $F_{i+1}$ for $i=1, \ldots, k-1$. Let $M_{j}^{(i)} D_{i+1}=M_{2 j-1}^{(i+1)} M_{2 j}^{(i+1)}$ for $j=1, \ldots, 2^{i}$, and $i=1, \ldots, k-1$.

(c) Each prime ideal of $D_{i}$ which lies over any of the prime ideals $p_{2} \mathbf{Z}, p_{3} \mathbf{Z}, \ldots p_{i+1} \mathbf{Z}$ in $\mathbf{Z}$ remains inertial with respect to $F_{i+1}$ for $i=1, \ldots, k-1$.

Now proceeding as in the proof of [3, Example 42.6], we obtain that there exists an algebraic integer $t_{k+1}$ of degree 2 over $F_{k}$ such that if $F_{k+1}=F_{k}\left(t_{k+1}\right)$, then $M_{j}^{(k)}$ decomposes with respect to $F_{k+1}$ for $j=1, \ldots, 2^{k}$ and each prime ideal of $D_{k}$ which lies over any of the prime ideals $p_{2} \mathbf{Z}, \ldots, p_{k+1} \mathbf{Z}$ in $\mathbf{Z}$ remains inertial with respect to $F_{k+1}$. Thus if $D_{k+1}$ denotes the integral closure of $\mathbf{Z}$ in $F_{k+1}$, then there exist prime ideals $M_{2 j-1}^{(k+1)}, M_{2 j}^{(k+1)}$ of $D_{k+1}$ such that $M_{j}^{(k)} D_{k+1}=M_{2 j-1}^{(k+1)} M_{2 j}^{(k+1)}$ for $j=1, \ldots, 2^{k}$. It follows as in the proof of [3, Example 42.6] that $\left\{M_{j}^{(k+1)}\right\}_{j=1}^{2^{k+1}}$ is the set of all prime ideals of $D_{k+1}$ which are lying over $p_{1} \mathbf{Z}$ in $\mathbf{Z}$ and moreover, it is clear from [3, Remark 41.9] that $D_{k+1} / M_{2 j-1}^{(k+1)} \cong D_{k} / M_{j}^{(k)}$ and $D_{k+1} / M_{2 j}^{(k+1)} \cong D_{k} / M_{j}^{(k)}$ as fields for $j=1, \ldots, 2^{k}$. As $D_{k} / M_{j}^{(k)}$ is isomorphic to $\mathbf{Z} / 2 \mathbf{Z}$ as fields for $j=1, \ldots, 2^{k}$, it follows that $D_{k+1} / M_{j}^{(k+1)} \cong \mathbf{Z} / 2 \mathbf{Z}$ as fields for $j=1, \ldots, 2^{k+1}$. Note that the conditions (a), (b), and (c) hold for each $i \in\{1, \ldots, k\}$.

It now follows by the principle of mathematical induction that there exist algebraic integers $\left\{t_{i}\right\}_{i=1}^{\infty}$ such that if $F_{i}=\mathbf{Q}\left(t_{1}, \ldots, t_{i}\right)$ and $D_{i}$ is the integral closure of $\mathbf{Z}$ in $F_{i}$, then conditions (a), (b), and (c) hold for each $i \in \mathbf{N}$.

Let $F=\cup_{i=1}^{\infty} F_{i}$ and $D=\cup_{i=1}^{\infty} D_{i}$. Observe that $D$ is integral over $\mathbf{Z}$ and hence $D$ is integral over $D_{k}$ for each $k \in \mathbf{N}$. Hence by [4, Theorem 48], $\operatorname{dim} D=\operatorname{dim} \mathbf{Z}=1$ and so any nonzero prime ideal of $D$ is a maximal ideal of $D$. We assert that there exist infinitely many maximal ideals of $D$ which are lying over $2 \mathbf{Z}$ in Z. Let $k \geq 1$. Since $D$ is integral over $D_{k}$, it follows from [1, Theorem 5.10] that there exists a prime ideal $P_{j}$ of $D$ such that $P_{j} \cap D_{k}=M_{j}^{(k)}$ for $j=1, \ldots, 2^{k}$. Since $M_{s}^{(k)} \neq M_{t}^{(k)}$ for any two distinct $s, t \in\left\{1, \ldots, 2^{k}\right\}$, it follows that $P_{s} \neq P_{t}$. Moreover, as $P_{j} \cap \mathbf{Z}=M_{j}^{(k)} \cap \mathbf{Z}=2 \mathbf{Z}$ for $j=1, \ldots, 2^{k}$, we obtain that for each $k \geq 1$, there exist at least $2^{k}$ prime ideals of $D$ which are lying over $2 \mathbf{Z}$ in $\mathbf{Z}$. This shows that there are infinitely many maximal ideals of $D$ which are lying over $2 \mathbf{Z}$ in $\mathbf{Z}$. Since $D$ is a one-dimensional integral domain and as 2 belongs to infinitely many maximal ideals of $D$, we obtain that $D$ does not have Noetherian spectrum. 
We now show that there are infinitely many maximal ideals $M$ of $D$ such that the multiplicatively closed subset $S=1+M$ of $D$ is saturated. Indeed, we show that if $M$ is any maximal ideal of $D$ with $M \cap \mathbf{Z}=2 \mathbf{Z}$, then $S=1+M$ is saturated. Let $M$ be any maximal ideal of $D$ such that $M \cap \mathbf{Z}=2 \mathbf{Z}$. Since $D=\cup_{k=1}^{\infty} D_{k}$, it follows that $M=\cup_{k=1}^{\infty}\left(M \cap D_{k}\right)$. As $2 \mathbf{Z}=M \cap \mathbf{Z}$, it follows that $\left(M \cap D_{k}\right) \cap \mathbf{Z}=2 \mathbf{Z}$ for each $k \geq 1$. Since $\left\{M_{j}^{(k)} \mid j \in\left\{1, \ldots, 2^{k}\right\}\right\}$ is the set of all prime ideals of $D_{k}$ which are lying over $2 \mathbf{Z}$ in $\mathbf{Z}$, it follows that $M \cap D_{k} \in\left\{M_{j}^{(k)} \mid j \in\left\{1, \ldots, 2^{k}\right\}\right\}$. Moreover, as $D_{k} / M_{j}^{(k)} \cong \mathbf{Z} / 2 \mathbf{Z}$ as fields for each $j=1, \ldots, 2^{k}$, we obtain that $D / M \cong \mathbf{Z} / 2 \mathbf{Z}$ as fields. This proves that $U(D / M)$ is trivial. Hence we obtain from Lemma 2.9 that the multiplicatively closed subset $S=1+M$ of $D$ is saturated. Since there are infinitely many maximal ideals of $D$ which are lying over $2 \mathbf{Z}$ in $\mathbf{Z}$, it follows that there are infinitely many maximal ideals $M$ of $D$ such that $S=1+M$ is saturated. Hence $D \notin \mathcal{R}$.

The above example also shows that the hypothesis that $R$ is semiquasilocal cannot be omitted in Proposition 4.3(i).

We mention in Example 4.8, a one-dimensional integral domain $R$ such that $R \in \mathcal{R}$ but $R$ does not have Noetherian spectrum. We make use of the following fact in the verification of Example 4.8.

Fact 4.7 Let $S$ be a saturated multiplicatively closed subset of a ring $T$ and let $R$ be a subring of $T$. Then the following hold:

(i) $S \cap R$ is a saturated multiplicatively closed subset of $R$.

(ii) If for some ideal I of $T$, the multiplicatively closed subset $S=1+I$ of $T$ is saturated, then the multiplicatively closed subset $1+(I \cap R)$ of $R$ is saturated.

Proof (i) This is obvious.

(ii) Since $(S=1+I) \cap R=1+(I \cap R)$, the proof of (ii) follows immediately from (i).

We next have the following example.

Example 4.8 We use the same notations as in [3, Example 42.6] except that we let $p_{1}=2$. Note that the domain $\mathbf{Z}^{*}$ given in [3, Example 42.6] is integral over $\mathbf{Z}$ and hence by [4, Theorem 48], $\operatorname{dim} \mathbf{Z}^{*}=\operatorname{dim} \mathbf{Z}=1$. Moreover, the integral domain $\mathbf{Z}^{*}$ constructed in [3, Example 42.6] is such that $p_{1}$ belongs to infinitely many maximal ideals of $\mathbf{Z}^{*}$. Hence $\mathbf{Z}^{*}$ does not have Noetherian spectrum. We verify below that $\mathbf{Z}^{*} \in \mathcal{R}$.

Let $\mathcal{C}$ denote the collection of ideals $I$ of $\mathbf{Z}^{*}$ such that the multiplicatively closed subset $S=1+I$ of $\mathbf{Z}^{*}$ is saturated. It is clear that $\mathbf{Z}^{*} \in \mathcal{C}$. Let $I$ be a proper ideal of $\mathbf{Z}^{*}$ such that $I \in \mathcal{C}$. Then it follows from Lemma 2.9 that $U\left(\mathbf{Z}^{*} / I\right)=\{\overline{1}\}$. Since $\{1,-1\} \subseteq U\left(\mathbf{Z}^{*}\right)$, it follows that $I \neq(0)$. With the notations being as in [3, Example 42.6], we know that $\mathbf{Z}^{*}=\cup_{i=1}^{\infty} \mathbf{Z}_{i}$, where $\mathbf{Z}_{i}$ denotes the integral closure of $\mathbf{Z}$ in $F_{i}=\mathbf{Q}\left(t_{1}, \ldots, t_{i}\right)$. Observe that $I=\cup_{k=1}^{\infty}\left(I \cap \mathbf{Z}_{k}\right)$. Now it follows from Fact 4.7(ii) that the multiplicatively closed subset $S_{k}=1+\left(I \cap \mathbf{Z}_{k}\right)$ of $\mathbf{Z}_{k}$ is saturated for each $k \in \mathbf{N}$ and the multiplicatively closed subset $1+(I \cap \mathbf{Z})$ of $\mathbf{Z}$ is saturated. As $\mathbf{Z}^{*}$ is integral over $\mathbf{Z}$ and $I$ is a nonzero ideal of $\mathbf{Z}^{*}$, we obtain that $I \cap \mathbf{Z}$ is a nonzero ideal of $\mathbf{Z}$. Since $I \neq \mathbf{Z}^{*}$, it is clear that $I \cap \mathbf{Z} \neq \mathbf{Z}$. Now it follows from Proposition 4.1 that $I \cap \mathbf{Z}=2 \mathbf{Z}$. Hence $\left(I \cap \mathbf{Z}_{k}\right) \cap \mathbf{Z}=I \cap \mathbf{Z}=2 \mathbf{Z}$ for $k=1,2, \ldots$. Moreover, since the multiplicatively closed subset $S_{k}=1+\left(I \cap \mathbf{Z}_{k}\right)$ of $\mathbf{Z}_{k}$ is saturated, it follows from Fact 4.2 that $I \cap \mathbf{Z}_{k}=\cap_{M} M$, where $M$ ranges through all the maximal ideals of $\mathbf{Z}_{k}$ with $M \supseteq I \cap \mathbf{Z}_{k}$ for $k=1,2, \ldots$ As $\left(I \cap \mathbf{Z}_{k}\right) \cap \mathbf{Z}=2 \mathbf{Z}$, we obtain that $M \cap \mathbf{Z}=2 \mathbf{Z}$ for each maximal ideal $M$ of $\mathbf{Z}_{k}$ with $M \supseteq I \cap \mathbf{Z}_{k}$. Let $\mathbf{A}_{k}$ denote the set of all maximal ideals of $\mathbf{Z}_{k}$ which are lying over $2 \mathbf{Z}$ in $\mathbf{Z}$. Note that in the notation of [3, Example 42.6], $\mathbf{A}_{k}=\left\{M_{1}^{(k)}, M_{2}^{(k)}, \ldots M_{k+1}^{(k)}\right\}$. Hence $I \cap \mathbf{Z}_{k}$ must be of the form $\cap_{M \in \mathbf{B}_{k}} M$, where $\mathbf{B}_{k}$ is some nonempty subcollection of $\mathbf{A}_{k}$. Now we obtain from the Chinese remainder theorem [1, Proposition 1.10(ii)] that $\mathbf{Z}_{k} / I \cap \mathbf{Z}_{k} \cong \prod_{M \in \mathbf{B}_{k}} \mathbf{Z}_{k} / M$. We know from Lemma 2.9 that $U\left(\mathbf{Z}_{k} / I \cap \mathbf{Z}_{k}\right)$ is trivial. Hence we obtain that $\mathbf{Z}_{k} / M \cong \mathbf{Z} / 2 \mathbf{Z}$ as fields for each $M \in \mathbf{B}_{k}$. It follows from the construction of [3, Example 42.6] that $\mathbf{B}_{k} \subseteq\left\{M_{k}^{(k)}, M_{k+1}^{(k)}\right\}$. Thus $I \cap \mathbf{Z}_{k} \in\left\{M_{k}^{(k)}, M_{k+1}^{(k)}, M_{k}^{(k)} \cap M_{k+1}^{(k)}\right\}$ and $I \cap \mathbf{Z}_{k+1} \in\left\{M_{k+1}^{(k+1)}, M_{k+2}^{(k+1)}, M_{k+1}^{(k+1)} \cap M_{k+2}^{(k+1)}\right\}$. Note that $I \cap \mathbf{Z}_{k}=\left(I \cap \mathbf{Z}_{k+1}\right) \cap \mathbf{Z}_{k}$. As $M_{k}^{(k)} \mathbf{Z}_{k+1}=M_{k}^{(k+1)}$ and $M_{k+1}^{(k)} \mathbf{Z}_{k+1}=M_{k+1}^{(k+1)} M_{k+2}^{(k+1)}$, it follows that $I \cap \mathbf{Z}_{k}=M_{k+1}^{(k)}$ for any $k \geq 1$. Hence we obtain that $I=\cup_{k=1}^{\infty}\left(I \cap \mathbf{Z}_{k}\right)=\cup_{k=1}^{\infty} M_{k+1}^{(k)}$. Moreover, if $I=\cup_{k=1}^{\infty} M_{k+1}^{(k)}$, then as $\mathbf{Z}_{k} / M_{k+1}^{(k)} \cong \mathbf{Z} / 2 \mathbf{Z}$ as fields for each $k \geq 1$, it follows that $\mathbf{Z}^{*} / I \stackrel{\mathrm{Z}}{\cong} \mathbf{2} \mathbf{Z}$ as fields. Hence we obtain from Lemma 2.9 that the multiplicatively closed subset $S=1+I$ of $\mathbf{Z}^{*}$ is saturated.

This proves that there are exactly two ideals $I$ of $\mathbf{Z}^{*}$ such that the multiplicatively closed subset $1+I$ of $\mathbf{Z}^{*}$ is saturated and they are $I=\mathbf{Z}^{*}$ and $I=\cup_{k=1}^{\infty} M_{k+1}^{(k)}$. Hence we obtain that $\mathbf{Z}^{*} \in \mathcal{R}$.

We next have the following proposition. 
Proposition 4.9 Let $K$ be an algebraic number field and let $R_{K}$ denote the ring of algebraic integers in $K$. Let $\mathcal{C}$ denote the collection of all ideals $J$ of $R_{K}$ such that the multiplicatively closed subset $1+J$ of $R_{K}$ is saturated. Then the following statements hold:

(i) $\mathcal{C}$ is finite.

(ii) If $J \in \mathcal{C}$ is such that $J \neq R_{K}$, then $J \cap \mathbf{Z}=2 \mathbf{Z}$.

Proof (i) Let $[K: \mathbf{Q}]=n$. We know from [2, Exercise 4.2.3], that $R_{K}$ is a free $\mathbf{Z}$-module of rank $n$. Thus $R_{K}$ is a finite integral extension domain of $\mathbf{Z}$. Hence $\operatorname{dim} R_{K}=1$ and $R_{K}$ is Noetherian. Since any Noetherian ring has Noetherian spectrum, we obtain from Proposition 4.5 that $\mathcal{C}$ is finite.

(ii) Let $J \in \mathcal{C}$ be such that $J \neq R_{K}$. Let $I=J \cap \mathbf{Z}$. We know from Fact 4.7(ii) that the multiplicatively closed subset $1+I$ of $\mathbf{Z}$ is saturated. Since $J \neq R_{K}$, it follows that $I \neq \mathbf{Z}$. Now it follows from Proposition 4.1 that $J \cap \mathbf{Z}=I=2 \mathbf{Z}$.

The following remark provides another proof of Proposition 4.9(i).

Remark 4.10 We remark here that Proposition 4.9(i) can also be proved as follows. Let $J \in \mathcal{C}$ be such that $J \neq R_{K}$. From Proposition 4.9(ii), we obtain that $J \cap \mathbf{Z}=2 \mathbf{Z}$ and so $J \supseteq 2 R_{K}$. We know from [2, Exercise 4.4.3] that for any nonzero ideal $A$ of $R_{K}, R_{K} / A$ is a finite ring. Hence it follows that $R_{K} / 2 R_{K}$ is a finite ring. Let $\left\{J_{1} / 2 R_{K}, J_{2} / 2 R_{K}, \ldots, J_{m} / 2 R_{K}\right\}$ be the collection of all ideals of $R_{K} / 2 R_{K}$. Note that $J / 2 R_{K}=J_{i} / 2 R_{K}$ for some $i \in\{1,2, \ldots, m\}$. Hence we obtain that $J=J_{i}$. This proves that $\mathcal{C}$ is finite.

We next provide some examples to illustrate Proposition 4.9.

Example 4.11 Let $d$ be a square-free integer. Let $K=\mathbf{Q}(\sqrt{d})$. Let $R_{K}$ denote the ring of algebraic integers in $K$. Let $\mathcal{C}$ denote the collection of all ideals $J$ of $R_{K}$ such that the multiplicatively closed subset $1+J$ of $R_{K}$ is saturated. Then the following hold:

(i) If $d \equiv 2$ or $3(\bmod 4)$, then $\mathcal{C}$ contains exactly two elements.

(ii) If $d \equiv 1(\bmod 8)$, then $\mathcal{C}$ contains exactly four elements and if $d \equiv 5(\bmod 8)$, then $R_{K}$ is the only element of $\mathcal{C}$.

Proof First note that it follows from Lemma 2.9 and Remark 2.5(ii) that any $J \in \mathcal{C}$ is a radical ideal of $R_{K}$.

(i) We know that $R_{K}=\mathbf{Z}[\sqrt{d}]$ [2, Example 4.3.4]. Suppose that $J \in \mathcal{C}$ and $J \neq R_{K}$. We know from Proposition 4.9(ii) that $J \cap \mathbf{Z}=2 \mathbf{Z}$. Note that $f(x)=x^{2}-d$ is the minimal polynomial of $\sqrt{d}$ over Q. Let $\bar{f}(x) \in(\mathbf{Z} / 2 \mathbf{Z})[x]$ be obtained by replacing the coefficients of $f(x)$ by their respective residue classes modulo 2 . We consider the following cases.

Case (a). $d \equiv 2(\bmod 4)$. Then $\bar{f}(x)=x^{2}$. Hence it follows from [2, Theorem 5.5.1] that $P=2 R_{K}+$ $\sqrt{d} R_{K}$ is a prime ideal of $R_{K}$ and moreover, $2 R_{K}=P^{2}$. Furthermore, $\left[R_{K} / P: \mathbf{Z} / 2 \mathbf{Z}\right]=1$. Since any nonzero prime ideal of $R_{K}$ is maximal, it follows from $2 R_{K}=P^{2}$ that $P$ is the only radical ideal of $R_{K}$ with the property that $P \cap \mathbf{Z}=2 \mathbf{Z}$. As $R_{K} / P \cong \mathbf{Z} / 2 \mathbf{Z}$, it follows that $U\left(R_{K} / P\right)=\{\overline{1}\}$ and so we obtain from Lemma 2.9 that $P \in \mathcal{C}$. Now it is clear from the above discussion that $P$ is the only element of $\mathcal{C}$ with $P \neq R_{K}$. Hence we obtain that $\mathcal{C}=\left\{R_{K}, P\right\}$.

Case (b). $d \equiv 3(\bmod 4)$. Then $\bar{f}(x)=x^{2}+\overline{1}=(x+\overline{1})^{2}$. Now it follows from [2, Theorem 5.5.1] that $N=2 R_{K}+(\sqrt{d}+1) R_{K}$ is a prime ideal of $R_{K}$ and $2 R_{K}=N^{2}$. Moreover, $\left[R_{K} / N: \mathbf{Z} / 2 \mathbf{Z}\right]=1$. As any nonzero prime ideal of $R_{K}$ is maximal, it follows from $2 R_{K}=N^{2}$ that $N$ is the only radical ideal of $R_{K}$ such that $N \cap \mathbf{Z}=2 \mathbf{Z}$. Since $R_{K} / N \cong \mathbf{Z} / 2 \mathbf{Z}$, it follows that $U\left(R_{K} / N\right)=\{\overline{1}\}$. Now we obtain from Lemma 2.9 that $N \in \mathcal{C}$. From the above discussion, it is clear that $\mathcal{C}=\left\{R_{K}, N\right\}$.

(ii) We know from [2, Example 4.3.4] that $R_{K}=\mathbf{Z}[(1+\sqrt{d}) / 2]$. Observe that $f(x)=x^{2}-x+(1-d) / 4$ is the minimal polynomial of $(1+\sqrt{d}) / 2$ over $\mathbf{Q}$. Let $\bar{f}(x) \in(\mathbf{Z} / 2 \mathbf{Z})[x]$ denote the polynomial obtained on replacing the coefficients of $f(x)$ by their respective residue classes modulo 2 . We need to consider the following two cases.

Case $(\mathbf{I}) . d \equiv 1(\bmod 8)$. Then $\bar{f}(x)=x^{2}-x=x(x+\overline{1})$. Now it follows from [2, Theorem 5.5.1] that $P_{1}=2 R_{K}+((1+\sqrt{d}) / 2) R_{K}$ and $P_{2}=2 R_{K}+(1+(1+\sqrt{d}) / 2) R_{K}$ are distinct prime ideals of $R_{K}$ with $2 R_{K}=P_{1} P_{2}$. Moreover, $\left[R_{K} / P_{1}: \mathbf{Z} / 2 \mathbf{Z}\right]=1=\left[R_{K} / P_{2}: \mathbf{Z} / 2 \mathbf{Z}\right]$. Observe that $R_{K} / P_{i} \cong \mathbf{Z} / 2 \mathbf{Z}$ for $i=1,2$ and so $U\left(R_{K} / P_{i}\right)$ is trivial for $i=1,2$. As $P_{1}+P_{2}=R_{K}$, it follows from the Chinese remainder theorem [1, Proposition 1.10(ii)] that $R_{K} / P_{1} \cap P_{2} \cong R_{K} / P_{1} \times R_{K} / P_{2} \cong \mathbf{Z} / 2 \mathbf{Z} \times \mathbf{Z} / 2 \mathbf{Z}$. 
Hence $U\left(R_{K} / P_{1} \cap P_{2}\right)$ is also trivial. Now it follows from Lemma 2.9 that $P_{1}, P_{2}, P_{1} \cap P_{2} \in \mathcal{C}$. Since $2 R_{K}=P_{1} P_{2}$, we obtain that $P_{1}, P_{2}, P_{1} \cap P_{2}$ are the only radical ideals $J$ of $R_{K}$ with $J \cap \mathbf{Z}=2 \mathbf{Z}$. Thus in this case we obtain that $\mathcal{C}=\left\{R_{K}, P_{1}, P_{2}, P_{1} \cap P_{2}\right\}$.

Case (II). $d \equiv 5(\bmod 8)$. Then $\bar{f}(x)=x^{2}+x+\overline{1}$. Note that $\bar{f}(x)$ is irreducible over $\mathbf{Z} / 2 \mathbf{Z}$. Now it follows from [2, Theorem 5.5.1] that $P=2 R_{K}$ is a prime ideal of $R_{K}$ and moreover $\left[R_{K} / P: \mathbf{Z} / 2 \mathbf{Z}\right]=2$. Observe that $P$ is the only proper radical ideal of $R_{K}$ with the property that $P \cap \mathbf{Z}=2 \mathbf{Z}$. As $\left[R_{K} / P\right.$ : $\mathbf{Z} / 2 \mathbf{Z}]=2$, it follows that $U\left(R_{K} / P\right)$ is not trivial. Hence we obtain that $P \notin \mathcal{C}$. Thus $R_{K}$ is the only element of $\mathcal{C}$.

Example 4.8 illustrates that the hypothesis that $R$ has Noetherian spectrum is not necessary in Proposition 4.5. In Example 4.13, we provide another example to illustrate it. We make use of the following fact in the verification of Example 4.13.

Fact 4.12 Let $\alpha=(1+\sqrt{5}) / 2$. Let $R=\mathbf{Z}[\alpha]$. Let $T$ be an extension ring of $R$. If $I$ is an ideal of $T$ such that the multiplicatively closed subset $S=1+I$ of $T$ is saturated, then $I=T$.

Proof Let $I$ be an ideal of $T$ such that the multiplicatively closed subset $S=1+I$ of $T$ is saturated. Since $R=\mathbf{Z}[\alpha]$ is a subring of $T$, it follows from Fact 4.7(ii) that the multiplicatively closed subset $1+(I \cap R)$ of $R$ is saturated. Hence we obtain from Example 4.11(ii) case (II) that $I \cap R=R$. Therefore, we obtain that $I=T$.

Example 4.13 Let $T=\{z \in \mathbf{C} \mid z$ is an algebraic integer $\}$ be the ring of all algebraic integers. Let $\mathcal{C}$ denote the collection of all ideals $I$ of $T$ such that the multiplicatively closed subset $S=1+I$ of $T$ is saturated. We assert that $T$ is the only element of $\mathcal{C}$. It is clear that $T \in \mathcal{C}$. Moreover, since $\mathbf{Z}[(1+\sqrt{5}) / 2]$ is a subring of $T$, it follows from Fact 4.12 that $\mathcal{C}=\{T\}$. This shows that $T \in \mathcal{R}$.

Since $T$ is integral over $\mathbf{Z}$, it follows from [4, Theorem 48] that $\operatorname{dim} T=\operatorname{dim} \mathbf{Z}=1$. It is known that any nonzero nonunit of $T$ belongs to uncountably many maximal ideals of $T$ [3, Proposition 42.8(1)]. Hence it follows that $T$ does not have Noetherian spectrum.

\section{When does $R \in \mathcal{R}$ imply that $R[x] \in \mathcal{R}$ ?}

Let $\mathcal{R}$ be as mentioned in the beginning of Sect. 4. Let $R$ be a ring and let $R[x]$ (respectively $R[[x]]$ ) denote the polynomial (respectively the power series) ring in one variable over $R$. Let $T=R[x]$ or $R[[x]]$. We prove in Corollary 5.2 that $R \in \mathcal{R}$ if $T \in \mathcal{R}$. Moreover, we prove in Proposition 5.3 that $R \in \mathcal{R}$ if and only if $R[[x]] \in \mathcal{R}$. I do not know if $R \in \mathcal{R}$ implies that $R[x] \in \mathcal{R}$. However, it is noted in Remark 5.6 that if $R$ is any semiquasilocal ring, then $R[x] \in \mathcal{R}$. Moreover, if $R$ is an integral domain with $\operatorname{char}(R) \neq 2, \operatorname{dim} R=1$, and if $R$ has Noetherian spectrum, then it is proved in Proposition 5.7 that $R[x] \in \mathcal{R}$. We prove in Proposition 5.8 that for a Boolean ring $R, R \in \mathcal{R}$ if and only if $R \cong F_{1} \times \cdots \times F_{m}$ as rings for some $m \geq 1$, where $F_{i}=\mathbf{Z} / 2 \mathbf{Z}$ for $i=1, \ldots, m$ if and only if $R[x] \in \mathcal{R}$. It is proved in Corollary 5.9 that a von Neumann regular ring $R \in \mathcal{R}$ if and only if $R[x] \in \mathcal{R}$. First we have the following lemma.

Lemma 5.1 Let $R_{1}, R_{2}$ be commutative rings with identity. Let $\phi: R_{1} \rightarrow R_{2}$ be a surjective homomorphism of rings. If $R_{1} \in \mathcal{R}$, then $R_{2} \in \mathcal{R}$.

Proof Let $\mathcal{C}$ denote the collection of all ideals $I$ of $R_{1}$ such that the multiplicatively closed subset $1+I$ of $R_{1}$ is saturated. Let $\mathcal{D}$ denote the collection of all ideals $J$ of $R_{2}$ such that the multiplicatively closed subset $1+J$ of $R_{2}$ is saturated. Let $J \in \mathcal{D}$. We assert that $J=\phi(I)$ for some $I \in \mathcal{C}$. If $J=R_{2}$, then $J=R_{2}=\phi\left(R_{1}\right)$ and it is clear that $R_{1} \in \mathcal{C}$. Suppose that $J \neq R_{2}$. Since $\phi: R_{1} \rightarrow R_{2}$ is a surjective homomorphism of rings, it follows that $R_{1} / \phi^{-1}(J) \cong R_{2} / J$ as rings. Hence we obtain that $U\left(R_{1} / \phi^{-1}(J)\right) \cong U\left(R_{2} / J\right)$ as groups. Since $J \in \mathcal{D}$ and $J \neq R_{2}$, it follows from Lemma 2.9 that $U\left(R_{2} / J\right)=\{\overline{1}\}$, where $\overline{1}$ is the residue class of 1 modulo $J$. Therefore, we obtain that $U\left(R_{1} / \phi^{-1}(J)\right)=\{\overline{1}\}$ (here $\overline{1}$ is the residue class of 1 modulo $\left.\phi^{-1}(J)\right)$. Now it follows from Lemma 2.9 that $\phi^{-1}(J) \in \mathcal{C}$. Let $I=\phi^{-1}(J)$. Since $\phi$ is onto, it is clear that $J=\phi\left(\phi^{-1}(J)\right)=\phi(I)$. Thus it is shown that $\mathcal{D} \subseteq\{\phi(I) \mid I \in \mathcal{C}\}$. Since $R_{1} \in \mathcal{R}, \mathcal{C}$ must be a finite collection. Hence we obtain that the collection $\mathcal{D}$ is finite. This proves that $R_{2} \in \mathcal{R}$.

As an immediate consequence of Lemma 5.1, we have the following corollary.

Corollary 5.2 Let $R$ be a ring and let $T=R[x]$ or $R[[x]]$. If $T \in \mathcal{R}$, then $R \in \mathcal{R}$. 
Proof The mapping $\phi: T \rightarrow R$ given by $\phi(f(x))=f(0)$ for any $f(x) \in T$ is a surjective homomorphism of rings. Hence if $T \in \mathcal{R}$, then it follows from Lemma 5.1 that $R \in \mathcal{R}$.

We next have the following proposition.

Proposition 5.3 Let $R$ be a ring. Then $R \in \mathcal{R}$ if and only if $R[[x]] \in \mathcal{R}$.

Proof Let $\mathcal{C}$ denote the collection of all ideals $I$ of $R$ such that the multiplicatively closed subset $1+I$ of $R$ is saturated. Let $\mathcal{D}$ denote the collection of all ideals $J$ of $R[[x]]$ such that the multiplicatively closed subset $1+J$ of $R[[x]]$ is saturated. Suppose that $R \in \mathcal{R}$. Hence the collection $\mathcal{C}$ is finite. We prove that the collection $\mathcal{D}$ is finite.

Let $J \in \mathcal{D}$. Suppose that $J \neq R[[x]]$. We know from Fact 4.2 that $J=\cap_{N} N$, where $N$ ranges over all maximal ideals of $R[[x]]$ with $N \supseteq J$. It follows from [1, Exercise 5(iii), p. 11] that $x \in N$ for any maximal ideal $N$ of $R[[x]]$. Hence we obtain that $x \in J$. Therefore, the ideal $J=(J \cap R) R[[x]]+x R[[x]]$. Since $J \in \mathcal{D}$, it follows from Fact 4.7(ii) that $J \cap R \in \mathcal{C}$. Thus it is shown that if $J \in \mathcal{D}$, then $J=$ $(J \cap R) R[[x]]+x R[[x]]$ with $J \cap R \in \mathcal{C}$. Since $\mathcal{C}$ is finite, it follows that $\mathcal{D}$ is finite. Moreover, if $I$ is any ideal of $R$, then $R[[x]] / I R[[x]]+x R[[x]] \cong R / I$ as rings. Thus if $I \in \mathcal{C}$, then it follows from Lemma 2.9 and the above isomorphism that $I R[[x]]+x R[[x]] \in \mathcal{D}$. Hence the above proof indeed shows that $|\mathcal{C}|=|\mathcal{D}|$. Therefore, $R[[x]] \in \mathcal{R}$.

Suppose that $R[[x]] \in \mathcal{R}$. Then it is already shown in Corollary 5.2 that $R \in \mathcal{R}$.

We make use of the following lemma in the proof of Proposition 5.5.

Lemma 5.4 Let $R_{i}, i=1, \ldots, n$ be rings and let $R=R_{1} \times \cdots \times R_{n}$ be their direct product. Then $R \in \mathcal{R}$ if and only if $R_{i} \in \mathcal{R}$ for $i=1, \ldots, n$.

Proof Suppose that $R \in \mathcal{R}$. Since $R_{i}$ is a homomorphic image of $R$, it follows from Lemma 5.1 that $R_{i} \in \mathcal{R}$ for $i=1, \ldots, n$.

Conversely, assume that $R_{i} \in \mathcal{R}$ for $i=1, \ldots, n$. We prove that $R=R_{1} \times \cdots \times R_{n} \in \mathcal{R}$. Let $\mathcal{C}(R)$ denote the collection of all ideals $I$ of $R$ such that the multiplicatively closed subset $(1, \ldots, 1)+I$ of $R$ is saturated. Let $\mathcal{C}\left(R_{i}\right)$ denote the collection of all ideals $I_{i}$ of $R_{i}$ such that the multiplicatively closed subset $1+I_{i}$ of $R_{i}$ is saturated for $i=1, \ldots, n$. Since $R_{i} \in \mathcal{R}$, the collection $\mathcal{C}\left(R_{i}\right)$ is finite for $i=1, \ldots, n$. We prove that the collection $\mathcal{C}(R)$ is finite.

Let $I \in \mathcal{C}(R)$. Note that $I=I_{1} \times \cdots \times I_{n}$ for some ideal $I_{i}$ of $R_{i}$ for $i=1, \ldots, n$. Suppose that $I \neq R$. Then $I_{i} \neq R_{i}$ for at least one $i \in\{1, \ldots, n\}$. Let $A=\left\{i \in\{1, \ldots, n\} \mid I_{i} \neq R_{i}\right\}$. Note that $R / I \cong \prod_{i \in A} R_{i} / I_{i}$ as rings. Hence $U(R / I) \cong \prod_{i \in A} U\left(R_{i} / I_{i}\right)$ as groups. Since $I \in \mathcal{C}(R)$ with $I \neq R$, we obtain from Lemma 2.9 that $U(R / I)$ is trivial and hence $U\left(R_{i} / I_{i}\right)$ is trivial for each $i \in A$. Therefore, it follows from Lemma 2.9 that $I_{i} \in \mathcal{C}\left(R_{i}\right)$ for each $i \in A$. If $i \notin A$, then $I_{i}=R_{i}$ which is clearly in $\mathcal{C}\left(R_{i}\right)$. Thus $I_{i} \in \mathcal{C}\left(R_{i}\right)$ for $i=1, \ldots, n$ and so $I=I_{1} \times \cdots \times I_{n} \in \mathcal{C}\left(R_{1}\right) \times \cdots \times \mathcal{C}\left(R_{n}\right)$. This proves that $\mathcal{C}(R) \subseteq \mathcal{C}\left(R_{1}\right) \times \cdots \times \mathcal{C}\left(R_{n}\right)$. Proceeding as above, with the help of Lemma 2.9, it is easy to show that $\mathcal{C}\left(R_{1}\right) \times \cdots \times \mathcal{C}\left(R_{n}\right) \subseteq \mathcal{C}(R)$. Hence we obtain that $\mathcal{C}(R)=\mathcal{C}\left(R_{1}\right) \times \cdots \times \mathcal{C}\left(R_{n}\right)$. Since $\mathcal{C}\left(R_{i}\right)$ is a finite collection for $i=1, \ldots, n$, it follows that the collection $\mathcal{C}(R)$ is finite. This proves that $R \in \mathcal{R}$

In Remark 5.6, we deduce with the help of Proposition 5.5 that for any semiquasilocal ring $R, R[x] \in \mathcal{R}$.

Proposition 5.5 Let $\mathcal{C}$ denote the collection of all ideals $I$ of a ring $R$ such that the multiplicatively closed subset $1+I$ of $R$ is saturated. If $R \in \mathcal{R}$ and if each proper ideal $I \in \mathcal{C}$ is the intersection of a finite number of maximal ideals of $R$, then $R[x] \in \mathcal{R}$.

Proof Let $\mathcal{D}$ denote the collection of all ideals $J$ of $R[x]$ such that the multiplicatively closed subset $1+J$ of $R[x]$ is saturated. We prove that $\mathcal{D}$ is finite. Let $J \in \mathcal{D}$ be a proper ideal of $R[x]$. Note that $J \cap R \neq R$ and we know from Fact 4.7(ii) that $J \cap R \in \mathcal{C}$. Hence we obtain from Lemma 2.9 that $U(R / J \cap R)=\{\overline{1}\}$. Moreover, we know from Fact 4.2 that $J \cap R=\cap_{M} M$, where $M$ ranges over all the maximal ideals $M$ of $R$ such that $M \supseteq J \cap R$. By hypothesis, $J \cap R$ is the intersection of a finite number of maximal ideals of $R$. Hence there exist maximal ideals $M_{1}, \ldots, M_{n}$ of $R$ such that $J \cap R=\cap_{i=1}^{n} M_{i}$. Since $U(R / J \cap R)=\{\overline{1}\}$, it follows from the proof of Proposition 2.7 that $\left|R / M_{i}\right|=2$ for $i=1, \ldots, n$.

Note that $J \supseteq \cap_{i=1}^{n} M_{i}[x]$. For convenience, let us denote the ideal $\cap_{i=1}^{n} M_{i}[x]$ by $K$. Observe that $(R[x] / K) /(J / K) \cong \stackrel{R}{x}[x] / J$ as rings. Since $U(R[x] / J)$ is trivial, it follows that $U((R[x] / K) /(J / K))$ is trivial. Hence we obtain from Lemma 2.9 that $J / K \in \mathcal{E}$, where $\mathcal{E}$ denotes the collection of all ideals $B / K$ 
of $R[x] / K$ such that the multiplicatively closed subset $\overline{1}+B / K$ of $R[x] / K$ is saturated. We assert that the collection $\mathcal{E}$ is finite. Now it follows from the Chinese remainder theorem [1, Proposition 1.10(ii)] that $R[x] / K \cong \prod_{i=1}^{n} R[x] / M_{i}[x]$ as rings. We know from Proposition 4.5 that for any field $F$, the polynomial ring $F[x] \in \mathcal{R}$. Since for any maximal ideal $M$ of $R, R[x] / M[x]$ is isomorphic to $(R / M)[x]$ as rings, it follows that $R[x] / M[x] \in \mathcal{R}$. Hence we obtain that $R[x] / M_{i}[x] \in \mathcal{R}$ for each $i=1, \ldots, n$ (as $\left|R / M_{i}\right|=2$ for $i=1, \ldots, n$, it is easy to verify that the ideals $A_{i}$ of $R[x] / M_{i}[x]$ with the property that $\overline{1}+A_{i}$ is a saturated multiplicatively closed subset of $R[x] / M_{i}[x]$ are five in number and they are given by $M_{i}[x] / M_{i}[x],\left(M_{i}[x]+\right.$ $\left.x R[x]) / M_{i}[x],\left(M_{i}[x]+(x-1) R[x]\right) / M_{i}[x],\left(M_{i}[x]+x(x-1) R[x]\right) / M_{i}[x], R[x] / M_{i}[x]\right)$. Since $R[x] / K \cong$ $\prod_{i=1}^{n} R[x] / M_{i}[x]$ as rings, we obtain from Lemma 5.4 that $R[x] / K \in \mathcal{R}$. Hence $\mathcal{E}$ is finite.

Thus if $J \in \mathcal{D}$ with $J \neq R[x]$, then $J \supseteq(J \cap R)[x], J \cap R \in \mathcal{C}$, and moreover, $J /(J \cap R)[x]$ varies over a finite collection. Since $R \in \mathcal{R}, \mathcal{C}$ is a finite collection. Now it is clear from the above discussion that $\mathcal{D}$ is finite and so $R[x] \in \mathcal{R}$.

The following remark shows that for any semiquasilocal ring $R, R[x] \in \mathcal{R}$

Remark 5.6 Let $R$ be a semiquasilocal ring. Then $R[x] \in \mathcal{R}$.

Proof We know from Proposition 4.3(i) that $R \in \mathcal{R}$. Let $\mathcal{C}$ denote the collection of all ideals $I$ of $R$ such that the multiplicatively closed subset $1+I$ of $R$ is saturated. Since $R$ is semiquasilocal, it follows from Fact 4.2 that any proper ideal $I \in \mathcal{C}$ is the intersection of a finite number of maximal ideals of $R$. Now it follows from Proposition 5.5 that $R[x] \in \mathcal{R}$.

As an immediate consequence of Propositions 4.5 and 5.5, we next have the following proposition.

Proposition 5.7 Let $R$ be an integral domain with $\operatorname{char}(R) \neq 2$. If $\operatorname{dim} R=1$ and $R$ has Noetherian spectrum, then $R[x] \in \mathcal{R}$.

Proof Let $\mathcal{C}$ denote the collection of all ideals $I$ of $R$ such that the multiplicatively closed subset $1+I$ of $R$ is saturated. By hypothesis, $R$ is a one-dimensional integral domain with Noetherian spectrum. Hence it follows from Proposition 4.5 that $R \in \mathcal{R}$. Let $I \in \mathcal{C}$ with $I \neq R$. Hence from Lemma 2.9, we obtain that $U(R / I)$ is trivial. Since $\operatorname{char}(R) \neq 2$ by assumption, $\{1,-1\} \subseteq U(R)$. Hence $I \neq(0)$. As $R$ is an integral domain, $\operatorname{dim} R=1$, and $R$ has Noetherian spectrum, it follows that $I$ can be contained in only a finite number of maximal ideals of $R$. Since $I \in \mathcal{C}$, we obtain from Fact 4.2 that $I=\cap_{M} M$, where $M$ ranges over all the maximal ideals $M$ of $R$ with $M \supseteq I$. This shows that any proper ideal $I$ of $R$ with $I \in \mathcal{C}$ is the intersection of a finite number of maximal ideals of $R$. Now it follows from Proposition 5.5 that $R[x] \in \mathcal{R}$.

Let $R$ be a Boolean ring. We determine in the following proposition when $R \in \mathcal{R}$.

Proposition 5.8 Let $R$ be a Boolean ring. Then the following statements are equivalent:

(i) $R \in \mathcal{R}$.

(ii) $R \cong F_{1} \times \cdots \times F_{m}$ as rings for some $m \geq 1$, where $F_{i}=\mathbf{Z} / 2 \mathbf{Z}$ for $i=1, \ldots, m$.

(iii) $R[x] \in \mathcal{R}$, where $R[x]$ is the polynomial ring in one variable over $R$.

Proof (i) $\Rightarrow$ (ii) We know from (ii) $\Rightarrow$ (iv) of Proposition 2.11 that for any ideal $I$ of $R$, the multiplicatively closed subset $1+I$ of $R$ is saturated. By hypothesis, $R \in \mathcal{R}$. Hence, we obtain that $R$ can have only a finite number of ideals and so $R$ can admit only a finite number of maximal ideals. Let $\left\{M_{1}, \ldots, M_{m}\right\}$ denote the set of all maximal ideals of $R$. Since $R$ is a Boolean ring, $R$ is reduced and $\operatorname{dim} R=0$. Hence it follows that $\cap_{i=1}^{m} M_{i}=(0)$. Now we obtain using the Chinese remainder theorem [1, Proposition 1.10(ii)] that $R \cong R / M_{1} \times \cdots \times R / M_{m}$ as rings. Since $\left|R / M_{i}\right|=2$ for $i=1, \ldots, m$, it is clear that $R \cong F_{1} \times \cdots \times F_{m}$ as rings, where $F_{i}=\mathbf{Z} / 2 \mathbf{Z}$ for $i=1, \ldots, m$.

(ii) $\Rightarrow$ (iii) By (ii), $R \cong F_{1} \times \cdots \times F_{m}$ as rings, where $F_{i}=\mathbf{Z} / 2 \mathbf{Z}$ for $i=1, \ldots, m$. Hence we obtain that $R[x] \cong F_{1}[x] \times \cdots \times F_{m}[x]$ as rings. Since $F_{i}[x] \in \mathcal{R}$ for $i=1, \ldots, m$, it follows from Lemma 5.4 that $R[x] \in \mathcal{R}$.

(iii) $\Rightarrow$ (i) For any ring $R, R[x] \in \mathcal{R}$ implies that $R \in \mathcal{R}$ by Corollary 5.2. This proves (iii) $\Rightarrow$ (i).

Let $R$ be a von Neumann regular ring. As a consequence of Lemma 5.1, Propositions 5.5 and 5.8, we prove in the following corollary that $R \in \mathcal{R}$ if and only if $R[x] \in \mathcal{R}$.

Corollary 5.9 Let $R$ be a von Neumann regular ring. Then the following statements are equivalent: 
(i) $R \in \mathcal{R}$.

(ii) $R \in \mathcal{R}$ and if I is a proper ideal of $R$ such that the multiplicatively closed subset $1+I$ of $R$ is saturated, then $I$ is the intersection of a finite number of maximal ideals of $R$.

(iii) $R[x] \in \mathcal{R}$.

Proof (i) $\Rightarrow$ (ii) It is clear that $R \in \mathcal{R}$. Let $\mathcal{C}$ denote the collection of ideals $I$ of $R$ such that the multiplicatively closed subset $1+I$ of $R$ is saturated. Let $I \in \mathcal{C}$ with $I \neq R$. We know from Lemma 2.9 that $U(R / I)=\{\overline{1}\}$. Moreover, we know from Fact 4.2 that $I=\cap_{M} M$, where $M$ varies over all the maximal ideals $M$ of $R$ with $M \supseteq I$. Since $R$ is a von Neumann regular ring, it follows that $R / I$ is also von Neumann regular ring. Now it follows from [3, Exercise 29, p. 113] and $U(R / I)=\{\overline{1}\}$ that each element of $R / I$ is idempotent. Hence, we obtain that $R / I$ is a Boolean ring. Since $R \in \mathcal{R}$, it follows from Lemma 5.1 that $R / I \in \mathcal{R}$. Thus, the Boolean ring $R / I \in \mathcal{R}$ and hence we obtain from the proof of (i) $\Rightarrow$ (ii) of Proposition 5.8 that $R / I$ can admit only a finite number of maximal ideals (indeed,it follows from (i) $\Rightarrow$ (ii) of Proposition 5.8 that $R / I \cong F_{1} \times \cdots \times \times F_{m}$ as rings for some $m \geq 1$, where $F_{i}=\mathbf{Z} / 2 \mathbf{Z}$ for $\left.i=1, \ldots, m\right)$. Hence, $I$ is the intersection of a finite number of maximal ideals of $R$.

(ii) $\Rightarrow$ (iii) This follows immediately from Proposition 5.5.

(iii) $\Rightarrow$ (i) This follows from Corollary 5.2.

Acknowledgments I am very much thankful to the referees for their many useful and helpful suggestions. Also I am very much thankful to Professor Salah Eddine Kabbaj for his support.

Open Access This article is distributed under the terms of the Creative Commons Attribution License which permits any use, distribution, and reproduction in any medium, provided the original author(s) and the source are credited.

\section{References}

1. Atiyah, M.F.; Macdonald, I.G.: Introduction to Commutative Algebra. Addisom-Wesley Publishing Company, Reading (1969)

2. Esmonde, J.; Ram Murty, M.: Problems in Algebraic Number Theory, Graduate Texts in Mathematics, 190. Springer, New York (2000)

3. Gilmer, R.: Multiplicative Ideal Theory. Marcel-Dekker, New York (1972)

4. Kaplansky, I.: Commutative Rings. The University of Chicago Press, Chicago (1974)

5. Matsumura, H.: Commutative Ring Theory. Cambridge studies in advanced mathematics 8, Cambridge University Press, Cambridge (1986)

6. Ohm, J.; Pendleton, R.: Rings with Noetherian Spectrum. Duke Math. J. 35, 631-640 (1968) 